\title{
Neutrino Signatures From Young Neutron Stars
}

Luke F. Roberts and Sanjay Reddy

\begin{abstract}
After a successful core collapse supernova (CCSN) explosion, a hot dense proto-neutron star (PNS) is left as a remnant. Over a time of twenty or so seconds, this PNS emits the majority of the neutrinos that come from the CCSN, contracts, and loses most of its lepton number. This is the process by which all neutron stars in our galaxy are likely born. The emitted neutrinos were detected from SN 1987A and they will be detected in much greater numbers from any future galactic CCSN. These detections can provide a direct window into the properties of the dense matter encountered inside neutron stars, and they can affect nucleosynthesis in the material ejected during the CCSN. In this chapter, we review the basic physics of PNS cooling, including the basic equations of PNS structure and neutrino diffusion in dense matter. We then discuss how the nuclear equation of state, neutrino opacities in dense matter, and convection can shape the temporal behavior of the neutrino signal. We also discuss what was learned from the late time SN 1987A neutrinos, the prospects for detection of these neutrinos from future galactic CCSNe, and the effects these neutrinos can have on nucleosynthesis.
\end{abstract}

\section{Introduction}

A nascent neutron star (NS) is often left as the remnant of a successful CCSN. This young NS emits a copious number of neutrinos over the first few seconds of its life. During this time it is referred to as a PNS. Due to the high densities and temperatures encountered inside the PNS, neutrinos cannot freely escape but instead

Luke F. Roberts

NASA Einstein Fellow, TAPIR, California Institute of Technology, Pasadena, CA. e-mail: lrobertsetapir.caltech.edu

Sanjay Reddy

Institute for Nuclear Theory, University of Washington, Seattle, WA. e-mail: sareddy@uw . edu

INT-PUB-16-051 
must diffuse out over a period of about a minute (Burrows and Lattimer. 1986). This neutrino emission is powered by a large fraction of the gravitational binding energy released by taking the iron core of a massive star and transforming it into a NS $\left(2-5 \times 10^{53} \mathrm{ergs}\right)$ (Baade and Zwicky, 1934). After about a minute, neutrinos can escape freely, which demarcates the transition from PNS to NS. This qualitative picture of late PNS neutrino emission was confirmed when about thirty neutrinos were observed from supernova (SN) 1987A over a period of about fifteen seconds (Bionta et al, 1987, Hirata et al, 1987). If a CCSN were observed in our galaxy today, modern neutrino detectors would see thousands of events (Scholberg, 2012). The neutrino signal is shaped by the nuclear equation of state $(\mathrm{EoS})$ and neutrino opacities. Therefore, detection of galactic CCSN neutrinos would give a detailed window into the birth of NSs and the properties of matter at and above nuclear density.

In addition to direct neutrino detection, there are other reasons why understanding the properties of these late-time CCSN neutrinos is important. First, they can influence nucleosynthesis in CCSNe (Woosley et al, 1990). In particular, PNS neutrino emission almost wholly determines what nuclei are synthesized in baryonic material blown off the surface of PNSs (Woosley et al, 1994, Hoffman et al, 1997; Roberts et al 2010). Second, the integrated neutrino emission from CCSNe receives a large contribution from PNS neutrinos. Therefore, accurate models of PNS neutrino emission can contribute to understanding the diffuse $\mathrm{SN}$ neutrino background (Nakazato et al, 2015). Finally, the neutrino emission from the "neutrinosphere" of PNSs gives the initial conditions for the study of both matter-induced and neutrinoinduced neutrino oscillations (Duan et al, 2006). The rate of PNS cooling also has the potential to put limits on exotic physics and possible extensions of the standard model using data already in hand from SN 1987A (Keil et al, 1997; Pons et al, $2001 \mathrm{~b}$ a).

In this chapter, we discuss PNS cooling and the late time CCSN neutrino signal. In section 2, we focus on the basic equations of PNS cooling (section 2.1) and models of PNS cooling (sections 2.2 and 2.4. In sections 3, we discuss the various ingredients that shape the CCSN neutrino signal, the nuclear equation of state, neutrino opacities, and convection, respectively. Finally-in sections 4.1, 4.2, and 4.3we discuss the observable consequences of late time CCSN neutrinos. Throughout the article, we set $\hbar=c=1$.

\section{PNS Cooling}

Essentially, all of the energy that powers the neutrino emission during a CCSN comes from the gravitational binding energy released when taking the white dwarf like iron core of the massive progenitor star and turning it into a NS (Baade and Zwicky, 1934,, which is 


$$
E_{\mathrm{SN}} \sim \frac{3 G M_{\mathrm{pns}}^{2}}{5 r_{\mathrm{NS}}} \approx 3 \times 10^{53} \mathrm{erg}\left(\frac{M_{\mathrm{pns}}}{M_{\odot}}\right)^{2}\left(\frac{r_{N S}}{12 \mathrm{~km}}\right)^{-1} .
$$

The CCSN shock forms at an enclosed mass of $\sim 0.4 M_{\odot}$ and the material that is shock heated increases the effective PNS radius. This provides a reservoir of gravitational potential energy that can be converted into neutrinos. Therefore, around two thirds of the total energy, $E_{\mathrm{SN}}$ is available during the PNS cooling phase.

After the CCSN shock has passed through the PNS, the interior entropy varies between one and six $k_{b} /$ baryon. Peak temperatures between $30-60 \mathrm{MeV}$ are reached during PNS evolution, while the surface of the PNS has a temperature around $3-5 \mathrm{MeV}$. The interior of the PNS is comprised of interacting protons, neutrons, and electrons, at densities greater than a few times nuclear saturation density $\left(\rho_{s} \approx 2.8 \times 10^{14} \mathrm{~g} \mathrm{~cm}^{-3}\right)$ towards the center of the PNS. It is also possible that more exotic degrees of freedom are present in the inner most regions of the PNS (Prakash et al, 1997).

During core collapse, electron capture on heavy nuclei removes around $40 \%$ of the electrons from the core before neutrinos become trapped (Hix et al, 2003), leaving behind $Y_{e} \approx 0.3$ in the core. $Y_{e}$ is the number of electrons per baryon and is equal to the proton fraction by charge conservation. Although this constitutes a large portion of the initial lepton number of the core, a cold NS has an even lower total lepton number. The lepton number of the PNS is the total number of electrons plus electron neutrinos minus the number of positrons and electron antineutrinos, which is a conserved quantity. In a cold NS, the interactions $e^{-}+p \rightarrow v_{e}+n$ and $n \rightarrow \bar{v}_{e}+e^{-}+p$ are in equilibrium. Equating these rates and solving for the electron fraction results in $Y_{e} \sim 0.1$ for the densities encountered in the cores of NSs. Therefore, the PNS must"deleptonize" to become a NS, which requires losing a total lepton number of around

$$
N_{L} \approx 3.4 \times 10^{56}\left(\frac{M_{\mathrm{pns}}}{1.4 M_{\odot}}\right),
$$

which must be removed from the PNS by neutrinos.

Inside the PNS, a copious number of neutrinos of all flavors are produced and scattered by weak interactions involving both the baryons and the leptons present in the medium. The rate at which neutrinos leave the PNS and carry off energy and lepton number will depend on thermal neutrino mean free path inside the PNS with energy $\varepsilon_{v} \sim 60 \mathrm{MeV}$. Using a reference weak interaction neutrino cross-section (see section 3.1

$$
\sigma_{v}=\frac{4 G_{F}^{2} \varepsilon_{v}^{2}}{\pi} \approx 3 \times 10^{-40} \mathrm{~cm}^{2}\left(\frac{\varepsilon_{v}}{60 \mathrm{MeV}}\right)^{2},
$$

where, $G_{F}$ is the Fermi coupling constant, $\varepsilon_{V}$ is the neutrino energy. A naive estimate of the neutrino mean free path in the PNS is then

$$
\lambda_{v} \sim \frac{1}{\bar{n}_{b} \sigma_{v}} \approx 14 \mathrm{~cm}\left(\frac{\bar{\varepsilon}_{v}}{60 \mathrm{MeV}}\right)^{-2}\left(\frac{R_{\mathrm{pns}}}{12 \mathrm{~km}}\right)^{3}\left(\frac{M_{\mathrm{pns}}}{M_{\odot}}\right)^{-1},
$$


where $\bar{n}_{b}=3 M_{\mathrm{pns}} /\left(4 \pi R_{\mathrm{pns}}^{3} m_{b}\right)$ is the average baryon density of the PNS and $\bar{\varepsilon}_{v}$ is a characteristic energy for neutrinos inside the PNS. The neutrino mean free path is much smaller than the radius of the PNS, which is around $12 \mathrm{~km}$ once the shock heated mantle has cooled. Therefore, neutrinos must escape from the PNS diffusively and will be in thermal and chemical equilibrium with the baryons and electrons throughout most of the PNS.

\subsection{The Equations of PNS Cooling}

Generally, PNS evolution is a neutrino radiation hydrodynamics problem where general relativity is important. A number of simplifications to the general system of equations can be made. First, the PNS cooling time scale is much longer than the sound crossing time of the PNS. Therefore, PNSs are very close to being in hydrostatic equilibrium and spherical symmetric. With these approximations, the equations of PNS cooling become (see (Burrows and Lattimer, 1986; Pons et al, 1999, Roberts, 2012) for detailed derivations)

$$
\begin{aligned}
\frac{d P}{d r} & =-\frac{G\left(M_{g}+4 \pi r^{3} P\right)(\rho+P)}{r^{2} \Gamma^{2}} \\
\frac{d M_{g}}{d r} & =4 \pi r^{2} \rho \\
\frac{d N}{d r} & =\frac{4 \pi r^{2} n_{b}}{\Gamma} \\
\frac{d \alpha}{d P} & =-\frac{\alpha}{P+\rho} \\
\frac{d Y_{L}}{d t} & =-\frac{\partial\left(4 \pi \alpha r^{2} F_{L}\right)}{\partial N} \\
\frac{d Y_{e}}{d t} & =\alpha \frac{S_{N}}{n_{b}} \\
\frac{d\left(\left(\rho+\rho_{v}\right) / n_{b}\right)}{d t} & =-\alpha^{-1} \frac{\partial\left(4 \pi \alpha^{2} r^{2} H_{v}\right)}{\partial N}-\left(P+P_{V}\right) \frac{d(1 / n)}{d t} \\
\frac{d\left(\rho / n_{b}\right)}{d t} & =\alpha \frac{S_{E}}{n_{b}}-P \frac{d(1 / n)}{d t} .
\end{aligned}
$$

Equations 5, 6, 7 and 8 are just the relativistic equations of hydrostatic equilibrium, where $P$ is the pressure, $\rho$ is energy density of the background fluid, $\rho_{v}$ is the energy density of the neutrinos, $n_{b}$ is the baryon density, $M_{g}$ is the gravitational mass, $N$ is the enclosed baryon number, $r$ is the radius, $\Gamma=\sqrt{1-2 G M_{g} / r}$ and $\alpha$ is the lapse function. The lepton fraction is $Y_{L}=Y_{e}+Y_{v_{e}}$, where $Y_{e}$ is the number of electron per baryon and $Y_{v_{e}}=\left(n_{v_{e}}-n_{\bar{v}_{e}}\right) / n_{b}$ is the local net number of electron neutrinos per baryon. Equations 9 , and 11 describe conservation of lepton number and total internal energy in the PNS. Throughout most of the PNS, equations 10 and 12 are 
zero and can be neglected since the neutrino number and energy source functions, $S_{N}$ and $S_{E}$, rapidly bring the neutrinos into thermal equilibrium with the background fluid. The energy flux and lepton number fluxes are given by $F_{L}=F_{v_{e}}^{N}-F_{\bar{v}_{e}}^{N}$ and $H_{v}=\sum F_{i}^{E}$ (where the sum runs over all flavors of neutrinos and antineutrinos). The number and energy fluxes of individual neutrino species are given by

$$
F_{v_{i}}^{\{N / E\}}=\frac{2 \pi}{(2 \pi)^{3}} \int_{0}^{\infty} d \varepsilon \varepsilon^{\{2 / 3\}} \int_{-1}^{1} d \mu \mu f_{v_{i}}
$$

where $f_{v_{i}}=f_{v_{i}}(t, r, \varepsilon, \mu)$ is the distribution function of neutrinos of species $i, \mu$ is the cosine of the angle of neutrino propagation relative to the radial direction, and $\varepsilon$ is the neutrino energy. Below, we often discuss the neutrino luminosity, $L_{v_{i}}=$ $4 \pi r^{2} \alpha^{2} F_{i}^{E}$ and the neutrino number luminosity $\dot{N}_{v_{i}}=4 \pi r^{2} \alpha F_{i}^{N}$.

Solution of this system of equations requires a method of determining the $f_{v_{i}}$, the evolution of which is determined by the Boltzmann equation,

$$
\frac{D f_{v_{i}}}{D l}=\left(\eta_{a, i}+\eta_{s, i}\left[f_{v_{i}}\right]\right)\left(1-f_{v_{i}}\right)-\left(\kappa_{a, i}+\kappa_{s, i}\left[f_{v_{i}}\right]\right) f_{v_{i}},
$$

where $D / D l$ is a Lagrangian derivative in phase space, et $a_{a}$ and $\eta_{s}$ are absorption scattering emissivities, and $\kappa_{a}$ and $\kappa_{s}$ are absorption and scattering opacities (Lindquist, 1966; Thorne, 1981). The evolution of the $f_{v}$ can be attacked directly with the Boltzmann equation, but neutrino transport simplifies greatly throughout most of the PNS. As was mentioned above, the neutrino mean free path inside the PNS is much shorter than the distance over which $n_{b}, T$, and $Y_{e}$ are changing. Therefore, the neutrino distribution functions are very close to thermal and the neutrinos propagate through the star diffusively. In the diffusion limit diffusion limit of the Boltzmann equation, the number and energy flux of neutrinos of species $i$ are given by opacity weighted radial derivatives of the neutrino density (Burrows and Lattimer, 1986, Pons et al, 1999)

$$
F_{v i}^{\{N / E\}}=-\frac{\Gamma}{\alpha^{\{3 / 4\}}} \int_{0}^{\infty} d \omega \frac{\omega^{\{2 / 3\}}}{3\left(\kappa_{a, i}^{*}+\kappa_{s, i}^{*}\right)} \frac{\partial f_{i, \mathrm{FD}}(\omega / \alpha)}{\partial r},
$$

where $f_{i, \mathrm{FD}}(\varepsilon)=\left[1+\exp \left(\varepsilon / T-\eta_{i}\right)\right]^{-1}$ is the Fermi-Dirac distribution for neutrinos of species $i$ with degeneracy parameter $\eta_{i}, \omega$ is the neutrino energy at infinity. Both electron neutrinos and antineutrinos rapidly reach chemical equilibrium with the nuclear medium via charged current neutrino interactions. Therefore, the electron neutrino chemical potential is $\mu_{v_{e}}=\mu_{e}+\mu_{p}-\mu_{n}$ and $\mu_{\bar{v}_{e}}=-\mu_{v_{e}}$. Because of the large mass of the $\mu$ and $\tau$ particles, no net $\mu$ or $\tau$ number is produced in the PNS and $\mu_{v_{\mu}}=\mu_{v_{\tau}}=0$. The quantity $\kappa_{a, i}^{*}$ is the total absorption opacity corrected for detailed balance and $\kappa_{s, i}^{*}$ is the scattering transport opacity (Pons et al, 1999; Burrows et al, 2006). These opacities have units of inverse length and are approximately $\kappa \sim n_{b} \sigma_{v}$. In the equilibrium diffusion limit, the isotropic parts of the neutrino distribution functions only depends on the local temperature, as well as the electron 
neutrino degeneracy factor, $\eta_{v_{e}}=\mu_{v_{e}} / T$, for electron neutrinos and antineutrinos. The chemical potentials of the $\mu$ and $\tau$ neutrinos are zero throughout the PNS due to the large masses of the $\mu$ and $\tau$ particles. With these assumptions, the total lepton and energy fluxes in the diffusion limit become

$$
\begin{gathered}
F_{L}=-\frac{\Gamma T^{2}}{\alpha 6 \pi^{2}}\left[D_{3} \frac{\partial(\alpha T)}{\partial r}+D_{2} \alpha T \frac{\partial \eta_{v_{e}}}{\partial r}\right] \\
H_{V}=-\frac{\Gamma T^{3}}{\alpha 6 \pi^{2}}\left[D_{4} \frac{\partial(\alpha T)}{\partial r}+D_{3} \alpha T \frac{\partial \eta_{v_{e}}}{\partial r}\right],
\end{gathered}
$$

where $D_{2}=D_{2}^{v_{e}}+D_{2}^{\bar{v}_{e}}, D_{3}=D_{3}^{v_{e}}-D_{3}^{\bar{\nu}_{e}}$, and $D_{4}=\sum_{i} D_{4}^{i}$ are diffusion coefficients. The single species diffusion coefficients are Rosseland mean opacities defined by

$$
D_{n}^{i}=\int_{0}^{\infty} d \varepsilon \frac{\varepsilon^{n} f_{i, \mathrm{FD}}\left(1-f_{i, \mathrm{FD}}\right)}{T^{n+1}\left(\kappa_{a, i}^{*}+\kappa_{s, i}^{*}\right)} .
$$

Only electron neutrinos and antineutrinos contribute to the lepton flux diffusion coefficients while all species contribute to the energy flux diffusion coefficients. Note that the diffusion coefficients will be $\sim \lambda_{v}\left(\left\langle\varepsilon_{v}\right\rangle\right)$, where $\left\langle\varepsilon_{v}\right\rangle$ is an average neutrino energy in the medium. This makes it clear that gradients in the temperature and $\mu_{v_{e}}$ in the PNS core, combined with the neutrino opacities, drive its deleptonization and cooling.

In addition to these structure and transport equations, a model for the dense matter encountered in the PNS, as well as the initial configuration of $s$ and $Y_{L}$ versus radius, is required to predict the PNS neutrino signal. The EoS - which determines $\rho, P$, and $\mu_{v_{e}}$ as a function of $n_{b}, T$, and $Y_{e}$ - and the neutrino diffusion coefficients depend strongly on the properties of matter at and above nuclear saturation density.

The equilibrium diffusion equations described above provide an excellent approximation to neutrino transport in the optically thick interior regions of the PNS, are useful for understanding the basic properties of PNS cooling, and have been used - along with flux limiters to prevent superluminal transport of energy and lepton number (Burrows and Lattimer, 1986) - in numerous works studying the cooling of PNSs (Burrows and Lattimer, 1986, Keil and Janka, 1995, Pons et al, 1999, Roberts et al, 2012). Nonetheless, they are not suited to describing neutrino transport near the surface where the neutrino mean free paths become large. They also cannot provide any information about the average energies of the neutrinos that emerge from the neutron star since they assume neutrinos are everywhere in thermal equilibrium with the background material. Therefore, some works have employed non-equilibrium, spectral neutrino transport at varying levels of sophistication (Woosley et al, 1994. Sumiyoshi et al, 1995, Hüdepohl et al., 2010; Fischer et al, 2010; Roberts et al, 2012; Nakazato et al, 2013). These methods all evolve the non-equilibrium distribution function of the neutrinos, $f_{v_{i}}$, at a large number of neutrino energies using the Boltzmann equation or some approximation thereof. 


\subsection{PNS Evolution}

Here, the evolution of a fiducial $1.42 M_{\odot}$ PNS model is described. PNS evolution has been modeled using numerical codes for almost thirty years (Burrows and Lattimer. 1986; Burrows, 1987, Sumiyoshi et al, 1995, Keil and Janka, 1995; Keil et al, 1995, Pons et al, 1999, 2001a a, Fischer et al, 2010; Hüdepohl et al., 2010, Roberts et al, 2012; Roberts, 2012, Nakazato et al, 2013). Although the fidelity to the underlying micro- and macro-physics has improved with time, the basic features of PNS cooling are still the same. The illustrative model discussed in this section was calculated using the multi-energy group neutrino transport method described in (Roberts et al, 2012). Even so, throughout most of the PNS this more realistic transport methods reduces to the diffusion equations described above.

This calculation began with a pre-SN model of a $15 M_{\odot}$ star from (Woosley and Weaver 1995). The evolution through core collapse, bounce, and the cessation of shock expansion was followed. Once the SN shock crossed an enclosed baryonic mass of $1.42 M_{\odot}$, material outside this region was excised from the grid to crudely simulate the CCSN explosion. Then this PNS core was evolved for one hundred seconds. This model is comparable to models found in (Hüdepohl et al. , 2010; Fischer et al, 2010). We used an EoS similar to the Lattimer-Swesty equation of state with incompressibility $K_{s}=220 \mathrm{MeV}$ (Lattimer and Douglas Swesty, 1991) and all neutrino-nucleon interactions were treated in the elastic limit. The evolution of the interior structure of the model is shown in figure 1 . The time evolution of various quantities in the center of the PNS as well as its energy and lepton number losses are shown in figure 2. These figures also show the evolution of the star during the preexplosion phase of the SN (see the chapter "Neutrino emission from supernovae" for a detailed discussion of the early time emission).

The initial structure left behind after deleptonization during core collapse and shock propagation through the core can be seen with the yellow lines in the panels showing the entropy and the lepton fraction in figure 1 . There is a low entropy, high lepton number core and a high entropy, low lepton number mantle. The transition point between these two phases is near the maximum temperature point in the PNS. After collapse and bounce, the SN shock formed near this transition point, so the mantle is shock heated and the core is not. The high entropy found in the mantle increases its pressure and thereby increases its radius and decreases its density relative to the core.

The initial core lepton fraction, $Y_{L}=0.33$, is set by the point during core collapse when neutrinos become trapped, which occurs when the core reaches a density near $10^{-4} \mathrm{fm}^{-3}$ (Hix et al 2003). This can be seen in the second and fourth panels of figure 2 Electron neutrinos are able to rapidly remove lepton number from the mantle, so this region has $\eta_{v_{e}} \approx 0$.

Once the SN shock begins to explode the star and accretion slows down or ceases, PNS neutrino emission proceeds in three phases: the mantle contraction phase, the deleptonization phase, and the thermal cooling phase. The basic features of these different phases can be seen in figures 1 and 2 


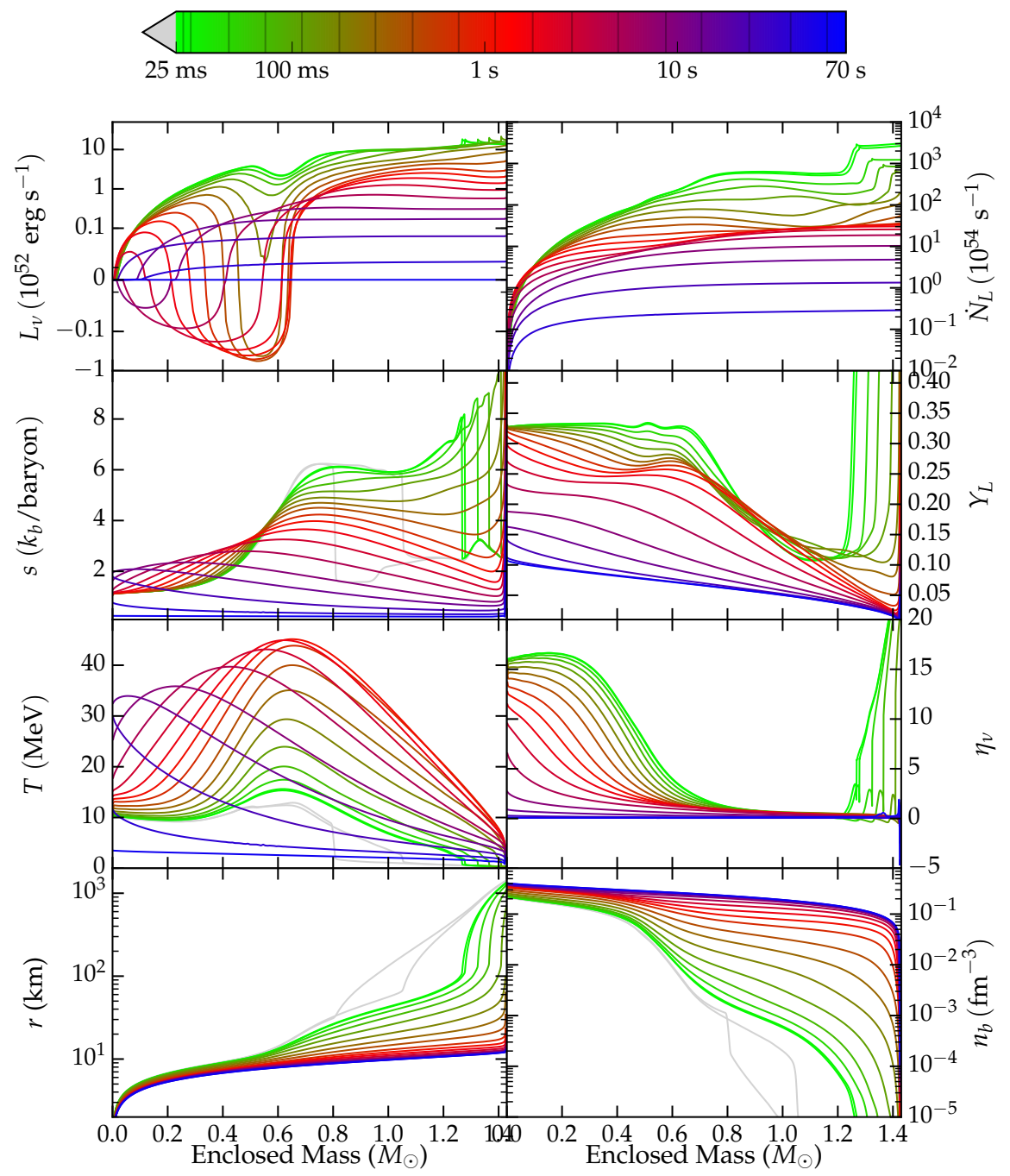

Fig. 1 Interior PNS quantities at selected times, with time coded by color. The semi-transparent lines on the color bar demarcate the time of the various lines shown in the panels below it. The gray lines are for selected times during the dynamical post-bounce evolution. Here, we focus on the evolution of the inner core after the dynamical phase has ended. The top left panel shows the energy carried by all flavors of neutrinos while the top right panel shows the net lepton number transported by neutrinos as a function of enclosed baryonic mass. The second row of panels shows the evolution of the entropy and lepton fraction, which is determined by the neutrino fluxes. The third row of panels shows the temperature and neutrino degeneracy parameter evolution. Gradients in these quantities drive the diffusive neutrino fluxes. The final row of panels shows the radius and baryon density as a function of enclosed baryonic mass to illustrate how the structure of the PNS evolves and contracts in response to loss of energy and lepton number. 


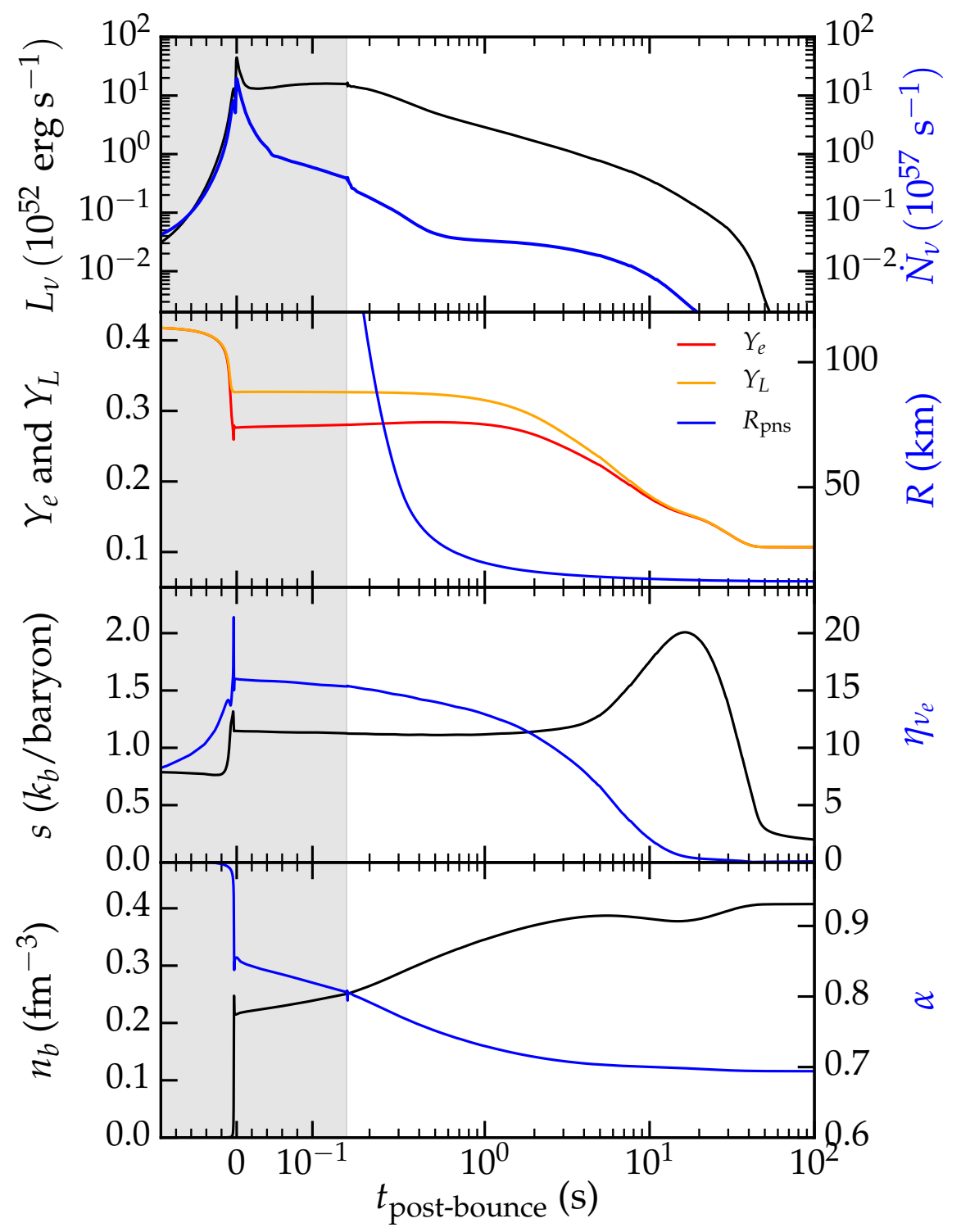

Fig. 2 Time evolution of central quantities and the total neutrino luminosity and lepton flux. The gray region corresponds to the accretion phase and is on a linear time scale, while the region to the right is the PNS cooling phase and it is plotted on a logarithmic scale. At the transition from the accretion phase to the PNS cooling phase, all of the material from above the shock is excised from the grid, causing a slight jump in some quantities. The top panel shows the total energy loss rate from the PNS and the deleptonization rate. The second panel shows the evolution of the central lepton fraction and electron fraction, as well as the PNS radius. The deleptonization era corresponds to the period over which $Y_{e}$ and $Y_{L}$ differ. The third panel shows the evolution of the central neutrino chemical potential and entropy. The impact of Joule heating is visible between five and twenty seconds. The bottom panel shows the central density and the central lapse, $\alpha$, to illustrate the contraction of the PNS over time. 
Mantle Contraction - During the first few seconds, the neutrino emission is dominated by contributions from the contracting mantle. This contraction is not dynamical. Rather, it is driven by the reduction in pressure due to entropy and electron losses. As can be seen in the second to last panel of figure 2, the radius of the PNS contracts from around $100 \mathrm{~km}$ to a value very close to the cold NS radius - which is $12 \mathrm{~km}$ for the EoS state used in this model - within the first two seconds of PNS evolution. The neutrino luminosity emerging from the surface of the PNS is sourced completely in this region, see the top left panel of figure 1 . In fact, energy is also being lost from the inner boundary of the mantle as electron antineutrinos and heavy flavored neutrinos diffuse down the positive temperature gradient into the core; the total neutrino luminosity becomes negative at the core mantle boundary. Additionally, the deleptonization wave pushes inward over this period but does not reach the center of the PNS. This can be seen in the panels of figure 1 showing $Y_{L}$ and $\eta_{v_{e}}$.

Deleptonization Phase - Once the PNS has settled down to close to the radius of a cold NS, the luminosity evolution is driven by the deleptonization wave that propagates into the center of the PNS and eventually brings the entire PNS to a state were $\eta_{v_{e}}=0$. This occurs over a period of about twenty seconds. The lepton number flux is produced by the negative gradient in $\eta_{v_{e}}$ left after shock breakout in the region between the homologous core and the base of the mantle.

During this period the core entropy and temperature increase, as can be seen in the bottom panel of figure 2 This is due to two effects. First, as was the case during the mantle contraction phase, electron antineutrinos and heavy flavored neutrinos are diffusing inward and heating the core. Second, lepton number is being lost from the core due to the positive flux of electron neutrinos throughout the PNS, which causes "Joule heating" (Burrows and Lattimer. 1986). To see this, we recast equation 11 in terms of the entropy using the first law of thermodynamics

$$
T \frac{d s}{d t}=-\alpha^{-1} \frac{\partial\left(4 \pi \alpha^{2} r^{2} H_{V}\right)}{\partial N}-\mu_{v_{e}} \frac{d Y_{L}}{d t} .
$$

Here, $s$ is the entropy per baryon in units of Boltzmann's constant, $T$ is the temperature, and $\mu_{v_{e}}$ is the electron neutrino chemical potential. Joule heating comes from the second term on the right hand side of this equation, since $d Y_{L} / d t<0$ and $\mu_{v_{e}}>0$. Eventually, the combined effects of these two processes raise the central entropy from around one to two $k_{b} /$ baryon and create a negative entropy and temperature gradients throughout the star.

Thermal Cooling Phase - Once $\eta_{v_{e}} \sim 0$ throughout the star (see figure1 1 , the PNS slowly contracts as energy leaks from the entire star. Both the entropy and lepton number of the core fall during this period, as shown in figure 2. The deleptonization rate falls off rapidly, but it does not go to zero because the local equilibrium electron fraction decreases with the local temperature, so low level deleptonization continues. The period of PNS cooling ends when the object becomes optically thin to neutrinos and the neutrino luminosity falls off abruptly. 


\subsection{Analytic Estimates of Cooling Phase Timescales}

It is instructive to use the equations of section 2.1 and a few simplifying assumptions to obtain analytic solutions to the neutrino transport equations. This can elucidate how the timescales of the deleptonization and thermal cooling phases depend on the neutrino opacities and the EoS.

At the onset of deleptonization electron neutrinos are degenerate. Under these conditions, the gradient in neutrino chemical potential dominates lepton number flux in Eq. 9 Further, due to Pauli blocking only neutrinos at the Fermi surface contribute and the relevant diffusion coefficient reduces to

$$
D_{2} \simeq \frac{\mu_{v_{e}}^{2}}{2 T^{2}} \frac{1}{\kappa_{a}^{*}\left(\mu_{v_{e}}\right)}
$$

In degenerate matter, as we shall show later in section 3.1

$$
\kappa_{a}^{*}\left(\mu_{v_{e}}, T\right) \approx \frac{G_{F}^{2}}{2 \pi} \frac{1+3 g_{A}^{2}}{4} M^{2} T^{2} \mu_{e} \simeq\left(\frac{k_{B} T}{15 \mathrm{MeV}}\right)^{2}\left(\frac{\mu_{e}}{200 \mathrm{MeV}}\right) \frac{1}{20 \mathrm{~cm}}
$$

where $M$ is the nucleon mass, $T$ is the temperature and $\mu_{e}$ is the electron chemical potential. Using Eq. 20 and neglecting general relativistic corrections we, can approximate the lepton number flux in Eq. 9 as

$$
F_{L} \approx \frac{n_{b}}{6 \kappa_{a}^{*}} \frac{\partial Y_{v_{e}}}{\partial r}
$$

By noting that $n_{b} / \kappa_{a}^{*}$ is a weak function of the density we ignore its spatial dependence to find an analytic solution of the separable form $Y_{v}(r, t)=Y_{v, 0} \phi(t) \psi(r)$ to Eq. 9. similar to the method descibed in (Prakash et al, 1997). Using appropriate boundary conditions at the surface we separately solve for spatial and temporal dependencies with $\phi(0)=1$ and $\psi(0)=1$. For the temporal part, which is of interest here, we obtain a simple exponential solution

$$
\phi(t)=\exp \left(\frac{-t}{\tau_{D}}\right) \quad \text { where } \quad \tau_{D} \simeq \frac{6\left\langle\kappa_{a}^{*}\right\rangle R^{2}}{C_{x}} \frac{\partial Y_{L}}{\partial Y_{V}} .
$$

Here, $\left\langle\kappa_{a}^{*}\right\rangle$ represents a spatial average of the charged current opacity inside the PNS and the constant $C_{x} \simeq 10$ depends on $\psi(r)$. Using fiducial values $T=15 \mathrm{MeV}$ and $\mu_{e}=200 \mathrm{MeV}$ and $\partial Y_{L} / \partial Y_{v}=5$ and setting $\left\langle\kappa_{a}^{*}\right\rangle=\kappa_{a}^{*}\left(\mu_{e}=200 \mathrm{MeV}, T=\right.$ $15 \mathrm{MeV}$ ) we obtain

$$
\tau_{D} \approx 11\left(\frac{R}{10 \mathrm{~km}}\right)^{2}\left(\frac{k_{B} T}{15 \mathrm{MeV}}\right)^{2}\left(\frac{\mu_{e}}{200 \mathrm{MeV}}\right)\left(\frac{\partial Y_{L}}{5 \partial Y_{v}}\right) s .
$$


This result, albeit arrived at with some approximation, clearly reveals the microphysics. The dependence on $T, \mu_{e}$, and $\partial Y_{L} / \partial Y_{v}$ is made explicit and we discuss later in section 3 how the dense matter EoS directly affects these properties.

We can also estimate the amount of Joule heating in the core (see equation 19 )

$$
\dot{E}_{\text {joule }}=-n_{b} \mu_{v} \frac{\partial Y_{L}}{\partial t} \approx n_{b} \mu_{v} \frac{\partial Y_{L}}{\partial Y_{v}} \frac{Y_{v, 0}}{\tau_{D}},
$$

where we have used Eq.23 to express the result in terms of the deleptonization time. For typical values of the deleptonization time $\tau_{D} \sim 11 \mathrm{~s}$, and $\partial Y_{L} / \partial Y_{V} \sim 5$, we find the heating rate per baryon $\dot{E}_{\text {joule }} / n_{b} \approx \mu_{v} Y_{v, 0} / 3$. At early times when $\mu_{v} \sim 150$ $\mathrm{MeV}$ and $Y_{v, 0} \sim 0.05$ the heating rate $\approx 2 \mathrm{MeV}$ per baryon per second will result in a similar rate of change in the matter temperature. This, coupled with the positive temperature gradients, results in a net heating of the inner core when $t<\tau_{D}$.

After deleptonization when the core begins to cool, the second term in Eq. 19 can be neglected and the energy flux

$$
H_{v} \approx \frac{T^{3}}{6 \pi^{2}} D_{4} \frac{\partial T}{\partial r} .
$$

Energy transport is dominated by $v_{\mu}, \bar{v}_{\mu}, v_{\tau}, \bar{v}_{\tau}$ and $\bar{v}_{e}$ neutrinos since their charged current reactions are suppressed and therefore they have larger mean free paths. For typical conditions where nucleons are degenerate and neutrino degeneracy is negligible, elastic neutral current scattering off nucleons is dominant source of opacity and (see section 3.1)

$$
\kappa_{s}^{*}\left(E_{v}\right) \simeq \frac{5}{6 \pi} G_{F}^{2} c_{A}^{2} \tilde{N}_{0} k_{B} T E_{v}^{2},
$$

where $\tilde{N}_{0}=\sum_{i=n, p} \partial n_{i} / \partial \mu_{i}$ is the effective density of nucleon states at the fermi surface to which neutrinos couple, and $c_{A} \simeq 1.2$ is the axial vector coupling. Using Eq. 27 the diffusion coefficient $D_{4}$ in Eq. 26 can be written as

$$
D_{4}=\frac{\pi^{3}}{G_{F}^{2} c_{A}^{2} \tilde{N}_{0}\left(k_{B} T\right)^{3}} .
$$

Substituting Eq. 28 in Eq. 26. Eq. 19 can be solved with the separable ansatz $T(r, t)=T_{c} \psi(x) \phi(t)$ to find a self-similar solution. We find that the temporal part $\phi(t)=1-\left(t / \tau_{c}\right)$, where

$$
\tau_{c} \approx \frac{2 \pi G_{F}^{2} c_{A}^{2}}{\beta}\left\langle N_{0} \frac{3 n_{b}}{\pi^{2}} \frac{\partial s}{\partial T}\right\rangle k_{B} T_{c} R^{2} \simeq 10 s \frac{k_{B} T_{c}}{30 \mathrm{MeV}} \frac{\left\langle n_{b}^{2 / 3}\right\rangle}{n_{0}^{2 / 3}}\left(\frac{R}{12 \mathrm{~km}}\right)^{2},
$$

where \langle\rangle denotes a spatial average, the numerical constant $\beta \cong 19$, and $n_{0}=0.16$ $\mathrm{fm}^{-3}$. Additionally, we have used $\partial s / \partial T=\pi^{2} N_{0} / 3 n_{b}$ and $N_{0}=M\left(3 \pi^{2} n_{b}\right)^{1 / 3} / \pi^{2}$, which hold for a non-relativistic, degenerate gas. The spatial averages and numerical value of $\beta$ are obtained by solving for the function $\psi(r)$. 


\subsection{PNS Neutrino Emission}

Here, we discuss the evolution of the flavor dependent neutrino luminosities and average energies, which constitute the detectable signal from the PNS. We focus on the properties of the neutrinos near the surface of the PNS. As the neutrinos propagate through the rest of the star, neutrino oscillations can change the flavor content of the neutrino fields and alter the spectra of the neutrinos that eventually reach earth (Duan et al, 2010). The effects of neutrino oscillations on the PNS signal are discussed in the chapter "Neutrino conversion in supernovae".

The evolution of the interior of the PNS drives the total energy and lepton number emitted from the surface, but the outermost layers of the PNS shape the spectrum of the emitted neutrinos. Therefore, it is convenient to describe the approximate radius at which neutrinos that escape to infinity are emitted, the "neutrino sphere" $R_{V}$. Following (Keil et al, 2003; Fischer et al, 2011), we define

$$
\tau_{\text {therm }}\left(R_{V}\right)=\int_{R_{V}}^{\infty} d r \sqrt{\left\langle\kappa_{a}\right\rangle\left(\left\langle\kappa_{a}\right\rangle+\left\langle\kappa_{s}\right\rangle\right)}=\frac{2}{3},
$$

where $\langle\kappa\rangle$ is an opacity averaged over the local neutrino distribution function. This is of course an approximation, since neutrino interactions have a strong energy dependence. Neutrinos of the same flavor but different energies will therefore decouple at different positions within the PNS. Nonetheless, the neutrino luminosity in a particular flavor can be reasonably estimated by assuming the neutrino sphere is a black-body emitter

$$
L_{V}=4 \pi \sigma_{B B} \phi R_{v}^{2} T\left(R_{V}\right)^{4},
$$

where $\sigma_{B B}=4.75 \times 10^{35} \mathrm{erg} \mathrm{MeV}^{-4} \mathrm{~cm}^{-2} \mathrm{~s}^{-1}$ is the black body constant and $\phi$ is a factor of order unity that accounts for deviations from strict Fermi-Dirac black body emission (Hüdepohl et al., 2010; Mirizzi et al, 2015). For a pure black body spectrum, the average energy of the emitted neutrinos would be $\left\langle\varepsilon_{V}\right\rangle \approx 3.15 T\left(R_{V}\right)$. The energy moments of neutrinos of species $i$ at radius $r$ are given by

$$
\left\langle\varepsilon_{v_{i}}^{n}\right\rangle=\frac{\int_{0}^{\infty} d \varepsilon \int_{-1}^{1} d \mu \varepsilon^{n+2} f_{v_{i}}}{\int_{0}^{\infty} d \varepsilon \int_{-1}^{1} d \mu \varepsilon^{2} f_{v_{i}}} .
$$

In reality, high energy neutrinos have a larger decoupling radius than lower energy neutrinos due to the approximate $\varepsilon_{v}^{2}$ scaling of the neutrino opacities (Keil et al, 2003). Therefore, high energy neutrinos are emitted from regions with lower temperatures. The emitted neutrino spectra then have a "pinched" character, where there is a deficit of high energy neutrinos relative to the Fermi-Dirac spectrum predicted by an energy averaged neutrinosphere (Janka and Hillebrandt, 1989).

The evolution of the neutrino sphere radii as a function of time are shown in the third panel and the temperature at the neutrino spheres is shown in the fourth panel of figure 3 . There are only small differences between the $\mu$ and $\tau$ flavored neutrino and antineutrino emission because they experience similar neutral current opacities. Therefore, we group all of these flavors together in flavor $x$. During the 


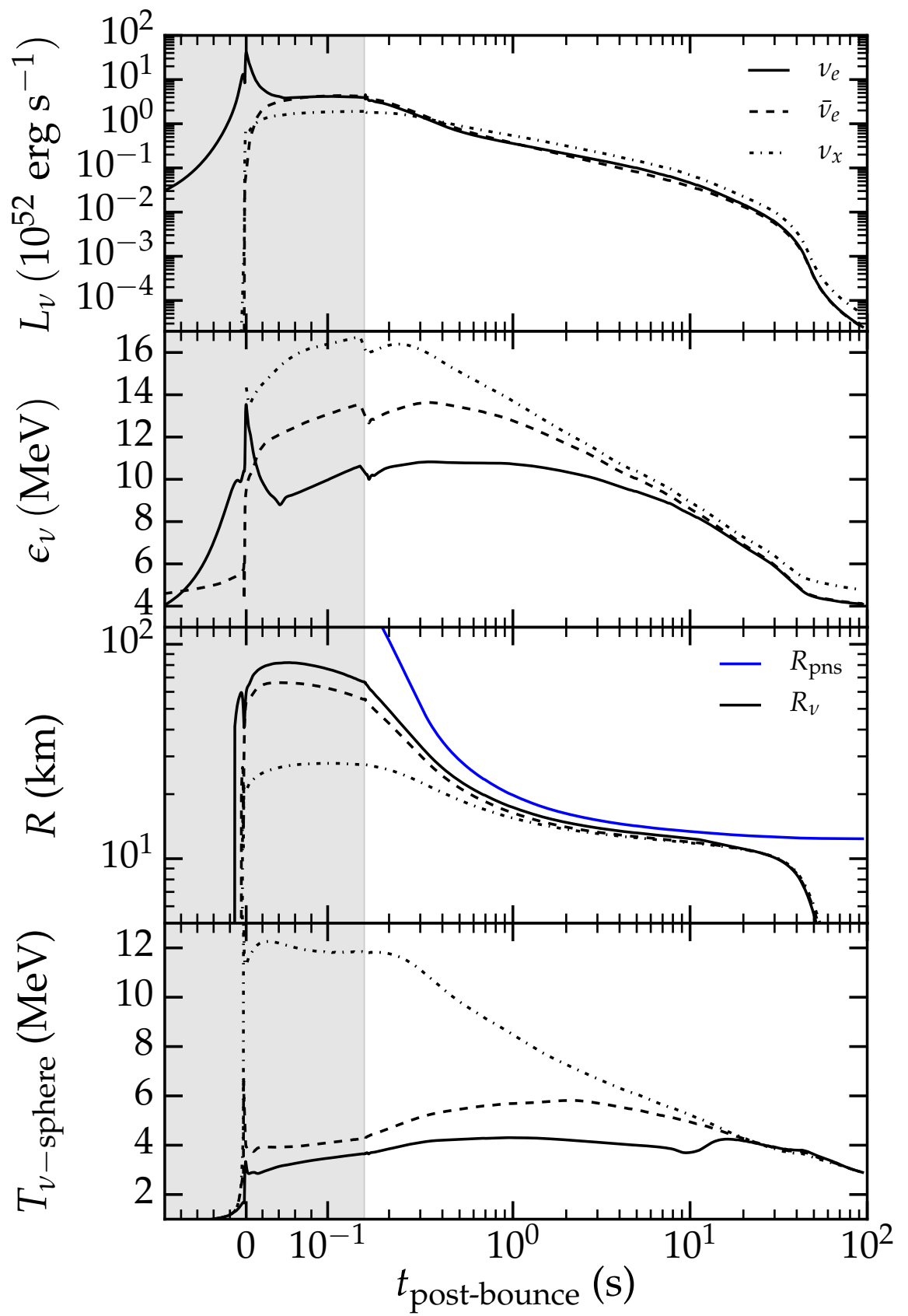

Fig. 3 Time evolution of neutrino properties at infinity and the properties of the neutrino spheres. The gray region corresponds to the accretion phase and is on a linear scale, while the region to the right is the PNS cooling phase and it is plotted on a logarithmic scale. We measure radiation quantities at a radius of $300 \mathrm{~km}$ or at the maximum radius of the simulation domain, whichever is smaller. At the transition from the accretion phase to the PNS cooling phase, all of the material from above the shock is excised from the grid, causing a slight jump in some quantities. In all of the panels, all heavy flavored neutrinos are represented by $v_{x}$, since their properties are all very similar. In the top panel, we show the neutrino luminosity for single flavors as a function of time. In the second panel, we show the evolution of the neutrino average energies. In the third panel we show the evolution of the neutrino sphere radii, along with the PNS radius, as a function of time. The simulation covers the entire time the PNS is optically thick to neutrinos. The bottom panel show the temperature at the neutrinospheres. Similar results can be found in (Fischer et al 2011). 
accretion phase and into the mantle contraction phase, there is a clear hierarchy with $R_{v_{x}}<R_{\bar{v}_{e}}<R_{v_{e}}$. This is driven mainly by differences in the charged current opacities: electron neutrinos get a large opacity contribution from the reaction $v_{e}+n \rightarrow e^{-}+p$ due to the large number of neutrons present near the decoupling region, electron antineutrinos get a somewhat smaller contribution from $\bar{v}_{e}+p \rightarrow e^{+}+n$ because of the small number of protons present in the decoupling region, and heavy flavored neutrinos receive no contribution to their opacity from charged current interactions. Since the temperature is decreasing with increasing radius, this gives rise to the standard early time hierarchy of neutrino energies $\left\langle\varepsilon_{v_{e}}\right\rangle\left\langle\left\langle\varepsilon_{\bar{v}_{e}}\right\rangle\left\langle\left\langle\varepsilon_{v_{x}}\right\rangle\right.\right.$ during the accretion and mantle cooling phases that can be seen in the second panel of figure 3 During this period, inelastic scattering from electrons outside of the neutrino sphere reduces the average energies of heavy flavored neutrinos relative to what the black body model would predict (Raffelt, 2001). In the deleptonization phase, there are very few protons in the outer layers of the PNS. Therefore, the opacities for the electron antineutrinos and the heavy flavored neutrinos are very similar and all of these neutrino flavors decouple at similar radii. In figure 3, these neutrinos have almost equal average energies over the majority of the PNS cooling phase. Eventually, during the thermal cooling phase all three neutrino spheres converge and the average emitted energies of all flavors become similar (Fischer et al, 2011), although the time at which they converge depends on how the charged current opacities are calculated (Roberts et al, 2012).

The top panel of 3 shows the luminosities of individual flavors of neutrinos. Soon after shock break out, the electron neutrino and antineutrino luminosities become very close to one another and stay similar throughout the entire cooling evolution. Deleptonization proceeds because the average energies of the electron neutrinos are lower and more electron neutrinos are required to carry a fixed luminosity than electron antineutrinos. Due to their small neutrinosphere, the heavy flavored neutrino luminosities are much lower than the electron neutrino luminosities during the mantle cooling phase. After mantle contraction, there is approximate equipartition of luminosity among the different flavors.

\section{Physics that shapes the cooling signal}

The PNS neutrino signal is interesting in part because it is shaped by the properties of neutron rich material at densities and temperatures that are inaccessible in the laboratory. Because of the high densities encountered throughout the PNS, the inter-nucleon separation is small enough that interactions between nuclei play a central role in determining the PNS EoS and the neutrino interaction rates. Over the past decade improved models to describe hot and dense matter were developed that reproduce empirically known properties of symmetric nuclear matter at saturation density. However, since matter encountered in the proto-neutron stars is characterized by a small proton fraction $x_{p} \simeq 0.05-0.3$, the symmetry energy defined through the relation 


$$
S\left(n_{b}\right)=E\left(n_{b}, x_{p}=1 / 2\right)-E_{n}\left(n_{b}, x_{p}=0\right),
$$

where $E\left(n_{b}, x_{p}=1 / 2\right)$ is the energy per particle of symmetric nuclear matter and $E\left(n_{b}, x_{p}=0\right)$ is the energy per particle of pure neutron matter, plays an important role. The energy of neutron-rich matter $E\left(n_{b}, x_{p}\right) \simeq E\left(n_{b}, x_{p}=1 / 2\right)+S\left(n_{b}\right)(1-$ $2 x_{p}^{2}$ ) since quartic and higher order terms are found to be small in most theoretical calculations. In this context, $a b$ intio calculations of the energy of neutron matter at sub-saturation density (Gandolfi et al, 2011, Tews et al, 2013) have provided valuable guidance in the development of a new suite of models for hot and dense matter which are also consistent with recent neutron star radii in the range $10-12 \mathrm{~km}$. These smaller radii are favored by recent modeling efforts to extract the radius of neutron stars from x-ray observations of of quiescent neutron star in low mass x-ray binaries and $\mathrm{x}$-ray burst (Steiner et al, 2013, Ozel et al, 2015). Properties of matter at and around nuclear saturation density, especially $S\left(n_{b}\right)$, can influence deleptonization and neutrino cooling timescales, convection and the neutrino spectrum (Sumiyoshi et al, 1995, Roberts et al, 2012, Roberts et al, 2012). PNS evolution is also sensitive to the thermal properties of degenerate dense matter as discussed in 2.3. and for a discussion of it we refer the reader to Refs. (Prakash et al, 1997, Constantinou et al, 2014, Rrapaj et al, 2015). In the next two subsections, we describe how nuclear interactions can affect neutrino opacities and convective instabilities inside the PNS, both of which can alter the PNS cooling timescale.

At densities near and below nuclear saturation density, PNS matter is only composed of protons, neutrons, and electrons, but at higher densities it is possible for more exotic material to be present. Hyperons-baryons containing strange quarkscan be produced in the interior of the PNS because the weak interaction does not conserve strangeness (Prakash et al, 1997). Quark matter (Steiner et al, 2001, Pons et al, 2001b) or Bose condensates (Prakash et al, 1997; Pons et al, 2000, 2001a) may also exist in the inner most regions of PNSs. In addition to altering the neutrino opacities, these new degrees of freedom serve to soften the nuclear EoS at high density and reduce the maximum neutron star mass. This can lead to "metastable" PNSs that emit neutrinos for tens of seconds before collapsing to a black hole $(\mathrm{BH})$ when more exotic material forms in their core and pressure support is reduced. BH formation would abruptly end the neutrino signal and is therefore directly observable (Pons et al, 2001b). We do not discuss these effects in any more detail here, but refer the reader to (Prakash et al, 1997).

\subsection{Neutrino opacities in dense matter}

The neutrino scattering opactities and thereby the diffusion coefficients defined in section 2.1 receive contributions from neutrino scattering, absorption, and pairproduction processes, as well as the inverses of the latter two. Scattering contributions come from the reactions 


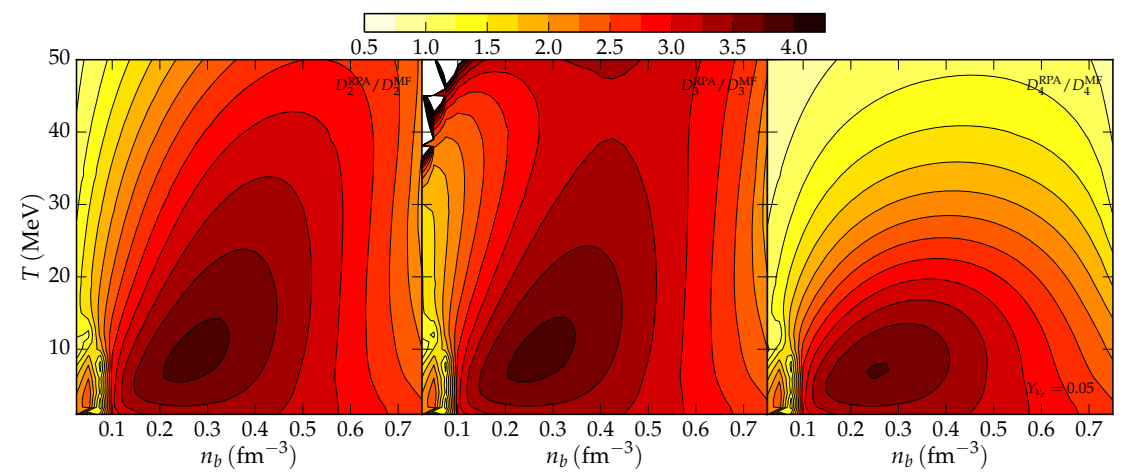

Fig. 4 Ratio of diffusion coefficients not including nuclear correlations to diffusion coefficients including nuclear correlations. At high density, weak charge screening (calculated using the random phase approximation) supresses the neutrino opacity and increases the neutrino diffusion coefficients. This reduces the PNS cooling timescale.

$$
\begin{aligned}
v_{i}+n & \rightleftharpoons v_{i}+n \\
v_{i}+p & \rightleftharpoons v_{i}+p \\
v_{i}+e^{-} & \rightleftharpoons v_{i}+e^{-} \\
v_{i}+e^{+} & \rightleftharpoons v_{i}+e^{+},
\end{aligned}
$$

as well as scattering from other possible components of the medium. All of the reactions above have neutral current contributions for all flavors of neutrinos, while $e^{-}$ and $e^{+}$scattering also have a charged current contribution for $v_{e}$ and $\bar{v}_{e}$ scattering, respectively. Since the dominant scattering contribution for all particles comes from the $n$ and $p$ scattering, there are only small differences between the scattering contributions to the diffusion coefficients for different neutrino flavors. Scattering from electrons and positrons can be highly inelastic, due to the small mass of the electron relative to the characteristic PNS neutrino energy, while scattering from neutrons and protons is close to elastic. This inelasticity can alter the emitted neutrino spectrum and serves to bring the average energies of the different neutrino species closer to one another (Hüdepohl et al., 2010).

The diffusion coefficients for the various neutrino flavors become different from one another due to charged current neutrino interactions. The main absorption contribution to $D_{i}^{v_{e}}$ comes from

$$
e^{-}+p \rightleftharpoons v_{e}+n
$$

while the main absorption contribution to $D_{i}^{\bar{v}_{e}}$ comes from

$$
e^{+}+n \rightleftharpoons \bar{v}_{e}+p
$$

All of the opacities receive contributions from thermal processes such as 


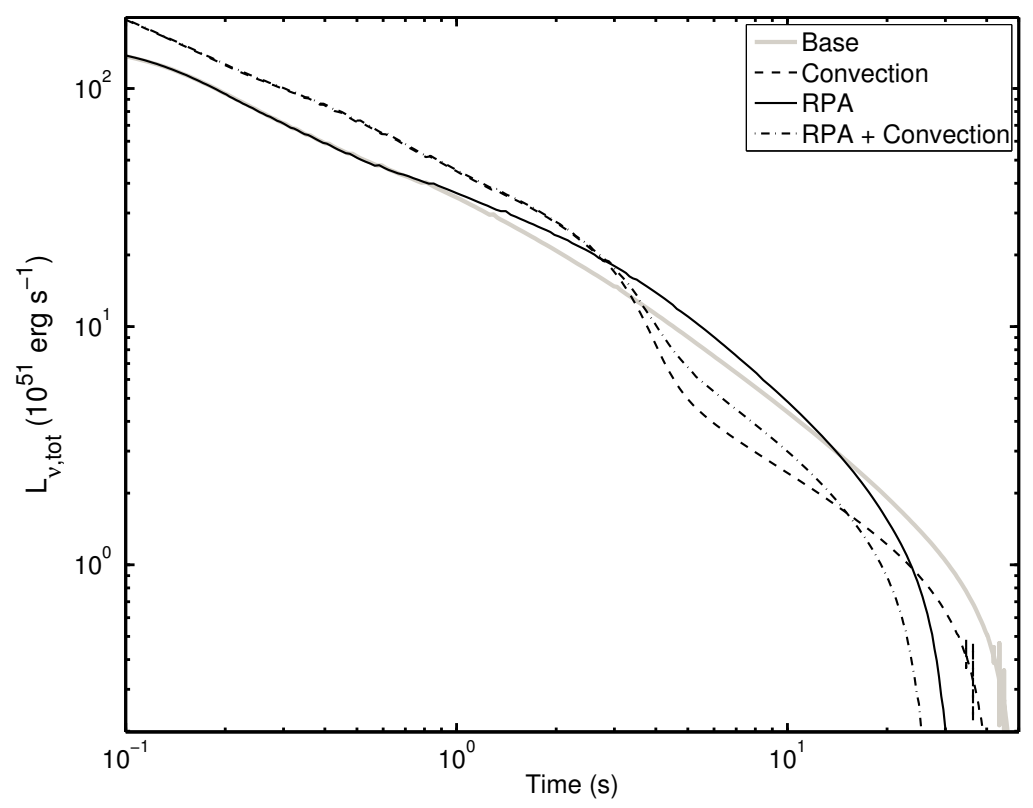

Fig. 5 The total PNS neutrino luminosity versus time for a number of PNS models that include convection and/or the affect of nuclear correlations on the opacity. Both convection and nuclear correlations decrease the cooling timescale relative to the baseline model. Convection alters the luminosity at early times, while correlations only become important after the mantle cooling phase. The models shown here are similar to those described in (Roberts et al, 2012).

$$
\begin{aligned}
N+N & \rightarrow N+N+v+\bar{v} \\
e^{-}+e^{+} & \rightarrow v+\bar{v}
\end{aligned}
$$

but these are usually small compared to the charged current interactions that affect the electron neutrinos and antineutrinos.

For both scattering and absorption processes, the cross section per unit volume for a general process $v+2 \rightarrow 3+4$ (where particle 3 is either a neutrino, electron, or positron) can be written as (Reddy et al, 1998)

$$
\kappa\left(\varepsilon_{v}\right)=\frac{2}{2 \varepsilon_{v}(2 \pi)^{9}} \int \frac{d^{3} p_{2}}{2 \varepsilon_{2}} \int \frac{d^{3} p_{3}}{2 \varepsilon_{3}} \int \frac{d^{3} p_{4}}{2 \varepsilon_{4}} f_{2}\left(\varepsilon_{2}\right)\left(1-f_{3}\left(\varepsilon_{3}\right)\right)\left(1-f_{4}\left(\varepsilon_{4}\right)\right)
$$




$$
\times(2 \pi)^{4} \delta^{4}\left(P_{v}+P_{2}-P_{3}-P_{4}\right)\left\langle|\mathscr{M}|^{2}\right\rangle,
$$

where

$$
\begin{aligned}
\left\langle|\mathscr{M}|^{2}\right\rangle= & 16 G_{F}^{2}\left[\left(C_{V}^{2}+C_{A}\right)^{2}\left(P_{v} \cdot P_{2}\right)\left(P_{3} \cdot P_{4}\right)+\left(C_{V}-C_{A}\right)^{2}\left(P_{2} \cdot P_{3}\right)\left(P_{V} \cdot P_{4}\right)\right. \\
& \left.-\left(C_{V}^{2}-C_{A}^{2}\right) M_{2} M_{4}\left(P_{v} \cdot P_{3}\right)\right]
\end{aligned}
$$

is the spin summed weak interaction matrix element of the process, $C_{V}$ and $C_{A}$ are vector and axial coupling constants, $\delta^{4}$ is the four-dimensional Dirac delta function, $P_{i}=\left(\varepsilon_{i},-\mathbf{p}_{i}\right)$ is the relativistic four-momentum, $p_{i}$ is the three-momentum, $\varepsilon_{i}$ is the energy, and $f_{i}$ is the distribution of species $i$. Particles 2 and 4 are always in thermal equilibrium inside PNSs, so $f_{2}$ and $f_{4}$ are isotropic Fermi-Dirac distribution functions. This expression comes from directly from Fermi's Golden Rule. If we specialize to particle 2 and 4 being nucleons, the reduced matrix element becomes independent of the nucleon momenta at leading order $v / c$ and the cross-section can be written as

$\kappa\left(\varepsilon_{v}\right)=\frac{G_{F}^{2}}{(2 \pi)^{2}} \int_{-1}^{1} d \mu_{3}\left(C_{V}^{2}\left(1+\mu_{3}\right)+C_{A}^{2}\left(3-\mu_{3}\right)\right) \int_{0}^{\infty} d \varepsilon_{3} p_{3} \varepsilon_{3}\left(1-f_{3}\left(\varepsilon_{3}\right)\right) S\left(q_{0}, q\right)$,

where $C_{V}$ and $C_{A}$ are weak vector and axial-vector coupling constants of the weak interaction, $\mu_{3}=\mathbf{p}_{v} \cdot \mathbf{p}_{3} /\left(\left|\mathbf{p}_{v}\right|\left|\mathbf{p}_{3}\right|\right)$, and

$$
S\left(q_{0}, q\right)=2 \int \frac{d^{3} p_{2}}{(2 \pi)^{3}} \int \frac{d^{3} p_{4}}{(2 \pi)^{3}} f_{2}\left(\varepsilon_{2}\right)\left(1-f_{4}\left(\varepsilon_{4}\right)\right)(2 \pi)^{4} \delta^{4}\left(Q+P_{2}-P_{4}\right)
$$

The energy-momentum transfer from the neutrino to the nucleons is denoted by the four-vector $Q=\left(q_{0},-\mathbf{q}\right)$, such that $\varepsilon_{3}=\varepsilon_{v}-q_{0}$ and $q=|\mathbf{q}|=\sqrt{\varepsilon_{v}^{2}+\varepsilon_{3}^{2}-2 \varepsilon_{v} \varepsilon_{3} \mu_{3}}$. This form of the opacity separates the contribution of the nucleons (or the "medium") from the neutrino and the outgoing particle (be it another particle or a neutrino with a different energy). The function $S\left(q_{0}, q\right)$ is often referred to as the response function or structure factor. A similar separation is found when the full momentum dependence of the matrix element is included, although there are multiple response functions with different kinematic dependence (Reddy et al, 1998). The contribution to the cross section from particle 3 is the amount of phase space available to it in the final state, which results in the leading order $\varepsilon_{v}^{2}$ dependence of weak interaction cross sections when $q_{0} \sim 0$.

The response function includes the effects of energy/momentum conservation, Pauli blocking of the final state nucleons, and thermal motion of the nucleons. When the momentum transfer $q$ is small (so that $\mathbf{p}_{2}=\mathbf{p}_{4}$ ), the response simplifies significantly. After manipulating the Fermi-Dirac distribution functions and integrating, one finds

$$
S\left(q_{0}, 0\right)=2 \pi \delta\left(q_{0}\right) \frac{n_{2}-n_{4}}{1-\exp \left(\left(\mu_{4}-\mu_{2}\right) / T\right)} .
$$


When species 2 and 4 are non-degenerate, this reduces to $2 \pi \delta\left(q_{0}\right) n_{2}$. This response is purely elastic, since $q_{0}=0$ and $\varepsilon_{v}=\varepsilon_{3}$. For scattering reactions, when species two equals species four, this response becomes

$$
S_{\text {scat }}\left(q_{0}, 0\right)=2 \pi \delta\left(q_{0}\right) T \frac{\partial n_{2}}{\partial \mu_{2}} .
$$

In the degenerate limit, the response can be shown to be (Reddy et al, 1998)

$$
S_{\operatorname{deg}}\left(q_{0}, q\right)=\frac{M^{2}\left(q_{0}+\mu_{2}-\mu_{4}\right)}{\pi q} \frac{\Theta\left(q-p_{F_{2}}+p_{F_{4}}\right)}{1-\exp \left(\left(\mu_{4}-\mu_{2}-q_{0}\right) / T\right)},
$$

where $p_{F_{i}}$ is the Fermi momentum of particles of species $i$ and $\Theta$ is the Heaviside step function. The opacity then becomes

$$
\kappa\left(\varepsilon_{v}\right)=\frac{G_{F}^{2}}{4 \pi^{3}}\left(C_{V}^{2}+3 C_{A}^{2}\right) M^{2} T^{2}\left(\varepsilon_{v}+\mu_{2}-\mu_{4}\right) \Xi \frac{\pi^{2}+\left(\frac{\varepsilon_{v}-\mu_{v}}{T}\right)^{2}}{1+\exp \left(\left(\mu_{v}-\varepsilon_{v}\right) / T\right)},
$$

where

$$
\Xi=\Theta\left(p_{F_{4}}+p_{F_{3}}-p_{F_{2}}-p_{F_{v}}\right)+\frac{p_{F_{4}}+p_{F_{3}}-p_{F_{2}}+p_{F_{v}}}{2 \varepsilon_{v}} \Theta\left(p_{F_{v}}-\left|p_{F_{4}}+p_{F_{3}}-p_{F_{2}}\right|\right) .
$$

When neutrinos are degenerate, only neutrinos near the Fermi surface will be able to scatter. The relevant opacity is then

$$
\kappa\left(\mu_{v}\right)=\frac{G_{F}^{2}}{8 \pi}\left(C_{V}^{2}+3 C_{A}^{2}\right) M^{2}\left(k_{B} T\right)^{2} \mu_{3},
$$

where we have assumed all four species are in equilibrium, $\mu_{v}+\mu_{2}=\mu_{3}+\mu_{4}$. These results are used in section 2.3 to estimate the deleptonization and thermal cooling timescales.

If nucleon-nucleon interactions are also considered, they can alter the response of the medium in a number of ways (Hannestad and Raffelt, 1998, Reddy et al, 1998, 1999: Burrows and Sawyer, 1998, Horowitz and Pérez-García. 2003). The simplest way to include nucleon-nucleon interactions is in the mean field approximation. In this approximation, the averaged interaction with all other nucleons gives single nucleons a momentum independent potential energy and an effective in medium mass. The nucleon energy-momentum relation then becomes $\varepsilon_{2,4}=p_{2,4}^{2} / 2 m_{2,4}^{*}+$ $U_{2,4}$. In the zero momentum transfer limit, the response becomes (Reddy et al, 1998)

$$
S_{\mathrm{MF}}\left(q_{0}, 0\right)=2 \pi \delta\left(q_{0}+\Delta U\right) \frac{n_{2}-n_{4}}{1-\exp \left(\left(\mu_{4}-\mu_{2}+\Delta U\right) / T\right)},
$$

where $\Delta U=U_{2}-U_{4}$. This implies that $\varepsilon_{3}=\varepsilon_{v}+\Delta U$. Because the cross-section strongly depends on the phase space available to particle 3 , a large, positive $\Delta U$ can increase the neutrino cross section while a negative $\Delta U$ will reduce the cross section (Martínez-Pinedo et al, 2012, Roberts et al, 2012). The potential energy of neutrons, 
$U_{n}$, differs from the potential energy of protons, $U_{p}$ due to the isospin dependence of the nuclear interaction. In neutron rich material, neutrons have a larger potential energy than protons because of the large, positive nuclear symmetry energy, $S\left(n_{b}\right)$, throughout the PNS. In fact, the potential energy difference can be related directly to the nuclear symmetry energy (Hempel, 2015). Therefore, mean field corrections to the response in PNSs increase the cross section for $v_{e}+n \rightarrow e^{-}+p$ and decrease the cross section for $\bar{v}_{e}+p \rightarrow e^{+}+n$. This change alters $D_{2}$ and $D_{3}$ and thereby the PNS deleptonization rate (Roberts, 2012). These corrections can also move the electron neutrino sphere to a larger radius and the electron antineutrinosphere to a smaller radius. This increases the difference between $\left\langle\varepsilon_{v_{e}}\right\rangle$ and $\left\langle\varepsilon_{\bar{v}_{e}}\right\rangle$, which may have large consequences for nucleosynthesis near the PNS (see section 4.3) (Roberts, 2012. Martínez-Pinedo et al, 2012).

When the neutrino wavelength is long compared to the inter-nucleon separation distance, neutrino interactions with the medium concurrently involve multiple nucleons at a microscopic level. In this limit, collective properties induced by nuclear interactions can significantly alter the response of the nuclear medium. The mean field approximation does not account for possible nucleon-nucleon correlations induced by interactions. Generally, accounting for these correlations is a complex many-body problem which has only been tackled within the random phase approximation (RPA) (Burrows and Sawyer, 1998; Reddy et al, 1999). The RPA essentially accounts for weak charge screening, which can reduce the neutrino opacity. In figure 4, we show the suppression of the diffusion coefficients by correlations. At high density, the corrections can be larger than a factor of two. This serves to decrease the cooling timescale of the PNS (Burrows and Sawyer, 1998, Reddy et al, 1999, Hüdepohl et al. 2010; Roberts et al, 2012). In figure 5, we show models of PNS cooling that include RPA corrections to the neutrino interaction rates compared with those that do not. During the mantle contraction phase they have little affect because the neutrino luminosity originates in a low density region. Once the neutrino luminosity is determined at higher densities, during the deleptonization and thermal cooling phases, these corrections decrease the neutrino emission timescale. The magnitude of this effect depends on the assumed nucleon-nucleon interaction (Keil et al, 1995: Reddy et al, 1999, Roberts et al, 2012).

\subsection{PNS Convection}

In addition to neutrinos, hydrodynamic motions of the PNS can transport energy and lepton number through the star. Although the majority of the PNS is in hydrostatic equilibrium, there can be regions which are unstable to the development of convection. Similar to the case in normal stellar burning, convective overturn can transport energy and lepton number much more rapidly than radiation and shorten the cooling timescale (Burrows, 1987, Wilson and Mayle, 1988, Roberts et al, 2012).

The standard Ledoux criterion for convective instability, adapted to the conditions found inside a PNS, is given by 


$$
\omega_{C}^{2}=-\frac{g}{\gamma_{n_{b}}}\left(\gamma_{s} \nabla \ln (s)+\gamma_{\gamma_{L}} \nabla \ln \left(Y_{L}\right)\right)>0,
$$

where $g$ is the local acceleration due to gravity and

$$
\gamma_{n_{b}}=\left(\frac{\partial \ln P}{\partial \ln n_{b}}\right)_{s, Y_{L}}, \gamma_{s}=\left(\frac{\partial \ln P}{\partial \ln s}\right)_{n_{b}, Y_{L}}, \gamma_{Y_{L}}=\left(\frac{\partial \ln P}{\partial \ln Y_{L}}\right)_{n_{b}, s} .
$$

These last three quantities are only functions of the nuclear EoS. In particular, $\gamma_{n_{b}}$ is related to the sound speed and $\gamma_{n_{b}}$ and $\gamma_{s}$ are always positive. The third thermodynamic derivative, $\gamma_{Y_{L}}$ can either be positive or negative; the pressure receives contributions from both electrons which have $\partial P_{e} / \partial Y_{e}>0$ and from the nucleons for which $\partial P_{N} / \partial Y_{e}<0$ when $Y_{e}<0.5$ due variations in $\partial S\left(n_{b}\right) / \partial n_{b}$ where $S\left(n_{b}\right)$ is the nuclear symmetry energy defined in Eq. 33 (Roberts et al, 2012). Noting that $\partial Y_{e} / \partial Y_{L}>0$ and of order unity, it is easy to see that the sign of $\gamma_{Y_{L}}$ can change depending on the relative contributions of electrons and nucleons. Therefore, the portion of the PNS that is convectively unstable depends on the assumed nuclear EoS and its symmetry energy, as well as the gradients of entropy and $Y_{L}$ (Roberts et al, 2012). The PNS may also be subject to doubly diffusive instabilities due to the lateral transport of composition and energy by neutrinos (Wilson and Mayle, 1988). These double diffusive instabilities would extend the region over which the PNS was unstable to hydrodynamic overturn.

The outer PNS mantle is unstable to adiabatic convection soon after the passage of the bounce shock (Epstein, 1979). This early period of instability beneath the neutrinospheres has been studied extensively in both one and two dimensions with the hope that it could increase the neutrino luminosities enough to lead to a successful CCSN explosion (Wilson and Mayle, 1988; Buras et al, 2006). During the deleptonization and thermal cooling phases, more and more of the PNS becomes unstable to convection because of the entropy and lepton gradients produced by neutrino cooling. Figure 1 shows that there are negative entropy gradients throughout the mantle for the entirety of the PNS phase and the negative gradient extends through the whole PNS by the end of deleptonization.

Because the cooling timescale is much longer than the dynamical timescale of the PNS, multi-dimensional simulations of late-time PNS convection have not been carried out to date. Rather, mixing length theory has been employed to study the impact of convection on the late time neutrino signal (Burrows, 1987, Roberts et al, 2012, Mirizzi et al, 2015). In figure 5, we show the total neutrino luminosity for models with and without convection to highlight the impact convection can have on the cooling timescale. At early times, the luminosity is elevated by around $25 \%$ due to convection until the period of convective instability ceases a few seconds after bounce. After this the luminosity is depressed relative to the case without convection, and the overall cooling timescale is reduced. 


\section{Observable Consequences}

\subsection{Neutrinos from SN 1987A}

Up to now, our discussion of PNS cooling has been mostly theoretical. In fact, the basics features of PNS cooling were reasonably well characterized before there was any observational evidence for this picture (Burrows and Lattimer. 1986). In February of 1987, both photons (Kunkel et al, 1987) and neutrinos (Bionta et al, 1987, Hirata et al, 1987; Alexeyev et al, 1988) reached Earth from a massive star that collapsed in the Large Magellanic Cloud, SN 1987A. Of course, the neutrinos are of the most interest for constraining PNS evolution, since the optical depth near the PNS is far too high for photons to escape this region. Bursts of sixteen, eight, and five neutrinos were observed near the time of SN 1987A in the Kamiokande (Hirata et al, 1987), IMB (Bionta et al, 1987), and Baksan (Alexeyev et al, 1988) neutrino detectors, respectively. Although there were only $\sim 30$ electron antineutrino events detected, they provided general confirmation of the previously developed theoretical picture of neutrino emission during core collapse and subsequent PNS cooling (Burrows and Lattimer, 1987). The neutrinos were observed within a 23 second window at Kamiokande and had energies ranging between 5 and $30 \mathrm{MeV}$. It is worth mentioning that this implies a PNS survived in the core of SN 1987A for at least twenty seconds, even though no central object has been observed in the remnant of SN 1987A to date (Graves et al, 2005).

In figure 6 , the joint probability distribution of the electron antineutrino spectral temperature and PNS cooling timescale inferred from the detected SN 1987A neutrino events by (Loredo and Lamb, 2002). They model the PNS cooling phase with a luminosity falling off with time as $L_{\bar{v}_{e}}(t) \propto\left(1+t / \tau_{c}\right)^{-4}$ and Fermi-Dirac neutrino spectrum with temperature $T_{\bar{v}_{e}}=T_{c, 0}\left(1+t / \tau_{c}\right)^{-1}$. The neutrino cooling timescale is best fit with $\tau_{c}=14.7 \mathrm{~s}$ and the antineutrino average energy at one second after bounce is best fit by $\left\langle\varepsilon_{\bar{v}_{e}}=13.7 \mathrm{MeV}\right.$. This is in reasonable qualitative agreement with the models in the literature and the one presented in section 2.4. It is quite an achievement that models of PNS cooling based on theoretical considerations alone before 1987 were able to reproduce the general features of the SN 1987A neutrino signal. Never the less, there is uncertainty in the cooling timescale and $T_{c, 0}$. Additionally, attempts have been made to constrain various physical processes operating during PNS cooling using the SN 1987A neutrino data (Burrows and Lattimer 1987, Keil et al, 1995, Reddy et al, 1999).

The neutrino cooling signal from SN 1987A has also been used to constrain beyond the standard model physics. Essentially, it is possible for exotic particles with very weak - but not too weak - interactions to rapidly remove energy from the PNS (Raffelt, 1996). If the amount of energy removed is comparable to the amount of energy emitted in neutrinos, the neutrino cooling timescale can be shortened. This technique has been mainly used to put limits on the properties of axions using the SN 1987A neutrino signal (Keil et al, 1997). 


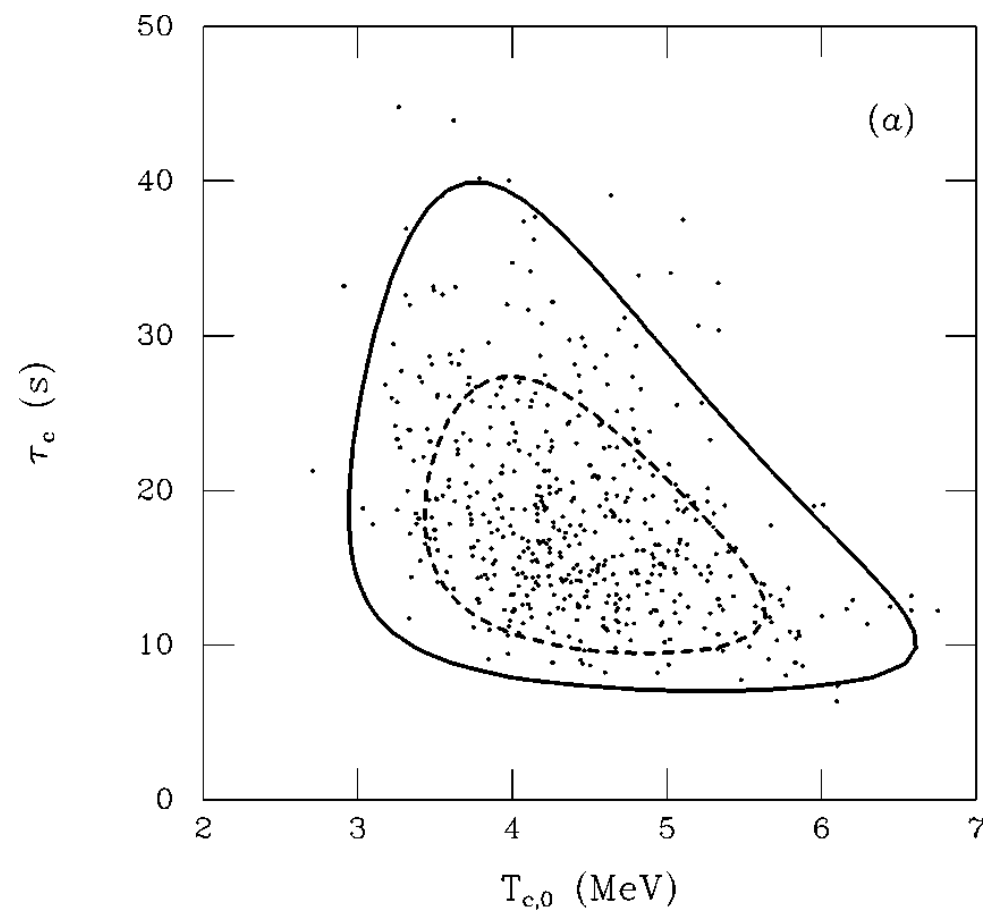

Fig. 6 Probability contours of the PNS cooling timescale, $\tau_{c}$, and the anti-electron neutrino spectral temperature $T_{c, 0}$ base on the SN 1987a neutrinos observed at the Super Kamiokande, Baksan, and IMB detectors, taken from (Loredo and Lamb, 2002). The dashed and solid lines demarcate regions of $68 \%$ and $95 \%$ credibility for the PNS cooling parameters, respectively. These inferred parameters are in good qualitative agreement with the predictions from models of PNS cooling. In this model, the average electron antineutrino energy is given by $\left\langle\varepsilon_{\bar{v}_{e}}\right\rangle \approx 3.15 T_{\bar{v}_{e}} \approx 13.7 \mathrm{MeV}$, where the last value is the value at one second post-bounce. Reprinted figure with permission from Loredo \& Lamb, Phys. Rev. D, Vol. 65, 063002, (2002). Copyright 2002 by the American Physical Society. 


\subsection{Galactic Supernova Neutrinos}

Any future galactic SN will yield far more neutrino detections than SN 1987A. The expected rate of CCSN in the Milky Way is around 1-2 per century (Cappellaro and Turatto, 2001), so direct CCSN detection is somewhat of a waiting game. Because of this, there are no dedicated CCSN neutrino detectors, but luckily there are many neutrino experiments that can moonlight as CCSN neutrino observatories.

The neutrino detection rate for a galactic CCSN can be found by integrating the neutrino distribution function at Earth over the response of the neutrino detector (Pons et al, 1999, Scholberg, 2012),

$$
\begin{aligned}
\frac{d N}{d t} & =\frac{2 \pi n_{d}}{(2 \pi)^{3}} \int_{0}^{\infty} d \varepsilon_{v} \varepsilon_{v}^{2} \sigma_{v}\left(\varepsilon_{v}\right) W\left(\varepsilon_{v}\right) \int_{-1}^{1} d \mu f_{v}\left(\varepsilon_{v}, \mu, D\right) \\
& \approx 87.5 \mathrm{~s}^{-1}\left(\frac{D}{10 \mathrm{kpc}}\right)^{-2}\left(\frac{\mathscr{M}_{\mathrm{det}}}{32 \mathrm{kt}}\right)\left(\frac{L_{\bar{v}_{e}}}{10^{51} \mathrm{erg} \mathrm{s}^{-1}}\right)\left(\frac{\left\langle\varepsilon_{\bar{v}_{e}}\right\rangle}{12 \mathrm{MeV}}\right) G\left[f_{\bar{v}_{e}}(\varepsilon)\right](49)
\end{aligned}
$$

where $D$ is the distance from Earth to the $\mathrm{SN}, n_{d}$ is the number of particles available to interact with neutrinos in the detector, and $W(\varepsilon)$ is the detector efficiency as a function of energy. In the second line, we estimate the electron antineutrino detection rate in a Cerenkov water detector with detector mass $\mathscr{M}$. The detector parameters chosen are meant to approximately correspond to the properties of SuperKamiokande (Fukuda et al, 2003). The dimensionless factor

$$
G\left[f_{v}(\varepsilon)\right]=\frac{m_{e}^{2} \int_{0}^{\infty} d \varepsilon \varepsilon^{2} \sigma_{v}(\varepsilon) W(\varepsilon) f_{v}}{\sigma_{v}\left(\varepsilon=m_{e}\right)\left\langle\varepsilon_{v}\right\rangle^{2} \int_{0}^{\infty} d \varepsilon \varepsilon^{2} f_{v}}
$$

encodes the spectral distribution of the neutrinos folded with the neutrino cross section and detector response. The presence of a detector threshold in $W(\varepsilon)$ can make the dependence of the count rate on the neutrino average energy steeper than is suggested by the simple scaling relation above. For a Fermi-Dirac distribution of neutrinos with zero chemical potential, $W$ has a value $\approx 1.8$. Integrating over a predicted CCSN neutrino signal, (Scholberg, 2012) find that around 7,000 electron antineutrino events would be detected in Super-Kamiokande for a SN $10 \mathrm{kpc}$ away and that the majority of these neutrinos would come from the PNS cooling phase (Mirizzi et al. 2015). Additionally, electron neutrinos will be detectable in liquid argon detectors through the reaction $v_{e}+{ }^{40} \mathrm{Ar} \rightarrow e^{-}+{ }^{40} \mathrm{~K}$. For a supernova at $10 \mathrm{kpc}$, we can expect about 700 events per kiloton (Scholberg, 2012). The $\simeq 40$ kiloton liquid Argon detector planned at the Deep Underground Neutrino Experiment (DUNE) will be able to provide valuable and complementary information about flavor and lepton number when combined with water Cerenkov detectors. Together, the large number of events and high energy resolution available from the current suite of neutrino experiments will put much more stringent constraints on the interior properties of PNSs when the next galactic CCSN is detected.

It is also possible that current neutrino detectors with upgrades or next generation neutrino detectors will be able to observe the diffuse background of neutrinos 
produced by CCSNe over the lifetime of the universe (Horiuchi et al, 2009). Predictions for the diffuse $\mathrm{MeV}$ scale neutrino background density depend on the integrated spectrum of neutrinos emitted during CCSNe, especially during the PNS cooling phase (Nakazato et al, 2015).

\subsection{Impact on CCSN Nucleosynthesis}

Neutrinos can alter the composition of material that is ejected from CCSNe, mainly in the innermost ejected regions (Woosley et al, 2002). The ejecta most affected by neutrinos is the material that comes from the surface of the PNS. Neutrino energy deposition in the atmosphere of the PNS can provide enough energy to unbind material from the surface and produce a neutrino driven wind (Duncan et al, 1986). Once outflowing material reaches large radii, the temperature drops and heavy nuclei form in the wind. The heating is driven by the charged current reactions

$$
\begin{aligned}
& v_{e}+n \rightarrow e^{-}+p \text { and } \\
& \bar{v}_{e}+p \rightarrow e^{+}+n .
\end{aligned}
$$

So, in addition to energizing the ejected material, neutrino interactions change its composition. The gravitational binding energy of a baryon at the surface of the PNS is $G M_{\mathrm{pns}} m_{N} / r_{\mathrm{pns}} \approx 160 \mathrm{MeV}$, so a baryon must undergo between ten and fifteen neutrino captures (given the expected average neutrino energies discussed above) to escape the potential well of the PNS. This number of interactions is large enough to push the material to a composition were electron neutrino capture balances electron antineutrino capture, which results in an electron fraction (Qian and Woosley, 1996)

$$
Y_{e, \mathrm{NDW}}=\frac{\lambda_{v_{e}}}{\lambda_{v_{e}}+\lambda_{\bar{v}_{e}}} .
$$

The neutrino capture rates are proportional to $\lambda_{v_{e}} \propto \dot{N}_{v_{e}}\left\langle\left(\varepsilon_{v_{e}}+\Delta_{n p}\right)^{2}\right\rangle / r^{2}$ and $\lambda_{\bar{v}_{e}} \propto \dot{N}_{\bar{v}_{e}} F_{\bar{v}_{e}}^{N}\left\langle\left(\varepsilon_{v_{e}}-\Delta_{n p}\right)^{2}\right\rangle / r^{2}$, where $\Delta_{n p}=1.293 \mathrm{MeV}$ is the neutron proton rest mass difference. Which heavy nuclei form depends very strongly on the electron fraction-as well as the entropy and dynamical timescale-of the outflowing material (Woosley et al, 1994, Sumiyoshi et al, 1995, Hüdepohl et al., 2010, Fischer et al, 2010; Roberts et al, 2012; Nakazato et al, 2013, Arcones and Thielemann, 2013). Therefore, the final composition depends on the difference between the average energies of electron neutrinos and antineutrinos. The magnitude of this difference in numerical models of PNS cooling is sensitive to the mean field corrections discussed in section 3.1. as well as to the method of neutrino transport (Hüdepohl et al. 2010, Fischer et al, 2010). It seems that the wind is at most marginally neutron rich, but this result depends on the properties of the assumed nuclear EoS (Roberts et al, 2012; Hempel, 2015, Mirizzi et al, 2015). 
The $v$-process is another mechanism by which PNS neutrinos can alter the composition of the material ejected from CCSNe (Woosley et al, 1990). Here, unlike in the neutrino driven wind, both charged current and neutral current neutrino interactions are responsible for altering the composition of the material. Therefore, neutrinos of all flavors contribute to the process. Essentially, the $v$-process alters the composition of ejected stellar material by the reactions

$$
\begin{aligned}
(Z, N)+v \rightarrow(Z, N)^{*}+v^{\prime} & \rightarrow(Z, N-1)+n+v^{\prime} \\
& \rightarrow(Z-1, N)+p+v^{\prime} \\
& \rightarrow(Z-2, N-2)+\alpha+v^{\prime}, \\
(Z, N)+v_{e} & \rightarrow(Z+1, N-1)+e^{-}, \text {and } \\
(Z, N)+\bar{v}_{e} & \rightarrow(Z-1, N+1)+e^{+},
\end{aligned}
$$

where $(Z, N)$ denotes a nucleus containing $Z$ protons and $N$ neutrons and $(Z, N)^{*}$ denotes an excitepd state of that nucleus. This is likely responsible for the production rare isotopes, such as ${ }^{11} \mathrm{~B},{ }^{19} \mathrm{~F},{ }^{15} \mathrm{~N}{ }^{138} \mathrm{La}$, and ${ }^{180} \mathrm{Ta}$, from much more common nuclei in the envelope of the SN. Additionally, it has been suggested that neutrons produced by neutrino interactions in the helium shell can be rapidly captured on preexisting heavy nuclei and make the r-process (Epstein et al, 1988), although later work has shown it is challenging to achieve the requisite conditions for this process (Banerjee et al, 2011). The nuclear yields produced by these process are sensitive to the time integrated flux and spectrum of neutrinos incident on the exterior material (Heger et al, 2005). Therefore, the properties of PNS neutrino emission, along with neutrino oscillations above the PNS, are very important to determining the results of $v$-process nucleosynthesis (Heger et al, 2005; Banerjee et al, 2011).

For further details on the impact of neutrinos on CCSNe nucleosynthesis, see the chapters "Effect of neutrinos on the ejecta composition of core collapse supernovae" and "Production of r-process elements in core collapse supernovae."

Acknowledgements We acknowledge our collaborators on this subject, including Gang Shen, Vincenzo Cirigliano, Jose Pons, Stan Woosley, and Ermal Rrapaj. LR was supported by NASA through an Einstein Postdoctoral Fellowship grant numbered PF3-140114 awarded by the Chandra X-ray Center, which is operated by the Smithsonian Astrophysical Observatory for NASA under contract NAS8-03060. SR was supported by the US Dept. of Energy Grant No. DE-FG0200ER41132.

\section{References}

Alexeyev EN, Alexeyeva LN, Krivosheina IV, Volchenko VI (1988) Detection of the neutrino signal from SN 1987A in the LMC using the INR Baksan underground scintillation telescope. Physics Letters B 205:209-214, DOI 10.1016/ 0370-2693(88)91651-6

Arcones A, Thielemann FK (2013) Neutrino-driven wind simulations and nu- 
cleosynthesis of heavy elements. Journal of Physics G Nuclear Physics 40(1):013201, DOI 10.1088/0954-3899/40/1/013201, 1207.2527

Baade W, Zwicky F (1934) On Super-novae. Proceedings of the National Academy of Science 20:254-259, DOI 10.1073/pnas.20.5.254

Banerjee P, Haxton WC, Qian YZ (2011) Long, Cold, Early r Process? NeutrinoInduced Nucleosynthesis in He Shells Revisited. Physical Review Letters 106(20):201104, DOI 10.1103/PhysRevLett.106.201104, 1103.1193

Bionta RM, Blewitt G, Bratton CB, Casper D, Ciocio A, Ciocio A, et al (1987) Observation of a neutrino burst in coincidence with supernova 1987A in the Large Magellanic Cloud. Physi Rev Lett 58:1494-1496, DOI 10.1103/PhysRevLett.58. 1494

Buras R, Rampp M, Janka H, Kifonidis K (2006) Two-dimensional hydrodynamic core-collapse supernova simulations with spectral neutrino transport. I. Numerical method and results for a 15 Mo star. Astron. \& Astrophys.447:1049-1092, DOI 10.1051/0004-6361:20053783, arXiv:astro-ph/0507135

Burrows A (1987) Convection and the mechanism of type II supernovae. Astrophys. J. Lett.318:L57-L61, DOI 10.1086/184937

Burrows A, Lattimer JM (1986) The birth of neutron stars. Astrophys. J. 307:178196, DOI 10.1086/164405

Burrows A, Lattimer JM (1987) Neutrinos from SN 1987A. Astrophys. J. Lett.318:L63-L68, DOI 10.1086/184938

Burrows A, Sawyer RF (1998) Effects of correlations on neutrino opacities in nuclear matter. Phys. Rev. C58:554-571, DOI 10.1103/PhysRevC.58.554, arXiv: astro-ph/9801082

Burrows A, Reddy S, Thompson TA (2006) Neutrino opacities in nuclear matter. Nuclear Physics A 777:356-394, DOI 10.1016/j.nuclphysa.2004.06.012, arXiv:astro-ph/0404432

Cappellaro E, Turatto M (2001) Supernova Types and Rates. In: Vanbeveren D (ed) The Influence of Binaries on Stellar Population Studies, Astrophysics and Space Science Library, vol 264, p 199, DOI 10.1007/978-94-015-9723-416, astro-ph/0012455

Constantinou C, Muccioli B, Prakash M, Lattimer JM (2014) Thermal properties of supernova matter: The bulk homogeneous phase. Phys Rev C89(6):065,802, DOI 10.1103/PhysRevC.89.065802, 1402.6348

Duan H, Fuller GM, Carlson J, Qian YZ (2006) Simulation of coherent nonlinear neutrino flavor transformation in the supernova environment: Correlated neutrino trajectories. Phys. Rev. D74(10):105014, DOI 10.1103/PhysRevD.74.105014, arXiv:astro-ph/0606616

Duan H, Fuller GM, Qian YZ (2010) Collective Neutrino Oscillations. Annual Review of Nuclear and Particle Science 60:569-594, DOI 10.1146/annurev.nucl. 012809.104524, 1001.2799

Duncan RC, Shapiro SL, Wasserman I (1986) Neutrino-driven winds from young, hot neutron stars. Astrophys. J. 309:141-160, DOI 10.1086/164587

Epstein RI (1979) Lepton-driven convection in supernovae. Mon. Not. Roy. Astro. Soc.188:305-325 
Epstein RI, Colgate SA, Haxton WC (1988) Neutrino-induced r-process nucleosynthesis. Physical Review Letters 61:2038-2041, DOI 10.1103/PhysRevLett. 61.2038

Fischer T, Whitehouse SC, Mezzacappa A, Thielemann FK, Liebendörfer M (2010) Protoneutron star evolution and the neutrino-driven wind in general relativistic neutrino radiation hydrodynamics simulations. Astron. \& Astrophys.517:A80, DOI 10.1051/0004-6361/200913106,0908.1871

Fischer T, Martínez-Pinedo G, Hempel M, Liebendörfer M (2011) Neutrino spectra evolution during proto-neutron star deleptonization. ArXiv e-prints 1112.3842

Fukuda S, Fukuda Y, Hayakawa T, Ichihara E, Ishitsuka M, Itow Y, et al (2003) The Super-Kamiokande detector. Nuclear Instruments and Methods in Physics Research A 501:418-462, DOI 10.1016/S0168-9002(03)00425-X

Gandolfi S, Carlson J, Reddy S (2011) The maximum mass and radius of neutron stars and the nuclear symmetry energy. ArXiv e-prints 1101.1921

Graves GJM, Challis PM, Chevalier RA, Crotts A, Filippenko AV, Fransson C, et al (2005) Limits from the Hubble Space Telescope on a Point Source in SN 1987A. Astrophys. J. 629:944-959, DOI 10.1086/431422, astro-ph/0505066

Hannestad S, Raffelt G (1998) Supernova Neutrino Opacity from Nucleon-Nucleon Bremsstrahlung and Related Processes. Astrophys. J. 507:339-352, DOI 10. 1086/306303, arXiv:astro-ph/9711132

Heger A, Kolbe E, Haxton WC, Langanke K, Martínez-Pinedo G, Woosley SE (2005) Neutrino nucleosynthesis. Physics Letters B 606:258-264, DOI 10.1016/ j.physletb.2004.12.017, arXiv:astro-ph/0307546

Hempel M (2015) Nucleon self-energies for supernova equations of state. Phys. Rev. C91(5):055807, DOI 10.1103/PhysRevC.91.055807, 1410.6337

Hirata K, Kajita T, Koshiba M, Nakahata M, Oyama Y, Sato N, et al (1987) Observation of a neutrino burst from the supernova SN1987A. Physical Review Letters 58:1490-1493, DOI 10.1103/PhysRevLett.58.1490

Hix WR, Messer OE, Mezzacappa A, Liebendörfer M, Sampaio J, Langanke K, et al (2003) Consequences of Nuclear Electron Capture in Core Collapse Supernovae. Physical Review Letters 91(20):201102, DOI 10.1103/PhysRevLett.91.201102, astro-ph/0310883

Hoffman RD, Woosley SE, Qian YZ (1997) Nucleosynthesis in Neutrino-driven Winds. II. Implications for Heavy Element Synthesis. Astrophys. J. 482:951-962, astro-ph/9611097

Horiuchi S, Beacom JF, Dwek E (2009) Diffuse supernova neutrino background is detectable in Super-Kamiokande. Phys. Rev. D79(8):083013, DOI 10.1103/ PhysRevD.79.083013, 0812.3157

Horowitz CJ, Pérez-García MA (2003) Realistic neutrino opacities for supernova simulations with correlations and weak magnetism. Phys. Rev. C68(2):025803, DOI 10.1103/PhysRevC.68.025803, arXiv:astro-ph/0305138

Hüdepohl et al L (2010) Neutrino Signal of Electron-Capture Supernovae from Core Collapse to Cooling. Physi Rev Lett 104(25):251,101-+, DOI 10.1103/ PhysRevLett.104.251101 
Janka HT, Hillebrandt W (1989) Monte Carlo simulations of neutrino transport in type II supernovae. Astron. \& Astrophys., Sup.78:375-397

Keil MT, Raffelt GG, Janka HT (2003) Monte Carlo Study of Supernova Neutrino Spectra Formation. Astrophys. J. 590:971-991, DOI 10.1086/375130, arXiv: astro-ph/0208035

Keil W, Janka HT (1995) Hadronic phase transitions at supranuclear densities and the delayed collapse of newly formed neutron stars. Astron. \& Astrophys.296:145-+

Keil W, Janka HT, Raffelt G (1995) Reduced neutrino opacities and the SN 1987A signal. Phys. Rev. D51:6635-6646

Keil W, Janka HT, Schramm DN, Sigl G, Turner MS, Ellis J (1997) Fresh look at axions and SN 1987A. Phys. Rev. D56:2419-2432, DOI 10.1103/PhysRevD.56. 2419, arXiv:astro-ph/9612222

Kunkel W, Madore B, Shelton I, Duhalde O, Bateson FM, Jones A, et al (1987) Supernova 1987A in the Large Magellanic Cloud. IAU Circular4316:1

Lattimer JM, Douglas Swesty F (1991) A generalized equation of state for hot, dense matter. Nuclear Physics A 535:331-376, DOI 10.1016/0375-9474(91) 90452-C

Lindquist RW (1966) Relativistic transport theory. Ann of Phys 37:487-518, DOI 10.1016/0003-4916(66)90207-7

Loredo TJ, Lamb DQ (2002) Bayesian analysis of neutrinos observed from supernova SN 1987A. Phys. Rev. D65(6):063,002-+, DOI 10.1103/PhysRevD.65. 063002

Martínez-Pinedo G, Fischer T, Lohs A, Huther L (2012) Charged-current weak interaction processes in hot and dense matter and its impact on the spectra of neutrinos emitted from proto-neutron star cooling. ArXiv e-prints 1205.2793

Mirizzi A, Tamborra I, Janka HT, Saviano N, Scholberg K, Bollig R, et al (2015) Supernova Neutrinos: Production, Oscillations and Detection. ArXiv e-prints 1508.00785

Nakazato K, Sumiyoshi K, Suzuki H, Totani T, Umeda H, Yamada S (2013) Supernova Neutrino Light Curves and Spectra for Various Progenitor Stars: From Core Collapse to Proto-neutron Star Cooling. Astrophys. J. Sup.205:2, DOI 10.1088/0067-0049/205/1/2, 1210.6841

Nakazato K, Mochida E, Niino Y, Suzuki H (2015) Spectrum of the Supernova Relic Neutrino Background and Metallicity Evolution of Galaxies. Astrophys. J. 804:75, DOI 10.1088/0004-637X/804/1/75, 1503.01236

Ozel F, Psaltis D, Guver T, Baym G, Heinke C, Guillot S (2015) The Dense Matter Equation of State from Neutron Star Radius and Mass Measurements. Submitted to Astrophys. J. 1505.05155

Pons JA, Reddy S, Prakash M, Lattimer JM, Miralles JA (1999) Evolution of ProtoNeutron Stars. Astrophys. J. 513:780-804, DOI 10.1086/306889, astro-ph/ 9807040

Pons JA, Reddy S, Ellis PJ, Prakash M, Lattimer JM (2000) Kaon condensation in proto-neutron star matter. Phys. Rev. C62(3):035803, DOI 10.1103/PhysRevC. 62.035803, nucl-th/0003008 
Pons JA, Miralles JA, Prakash M, Lattimer JM (2001a) Evolution of Proto-Neutron Stars with Kaon Condensates. Astrophys. J. 553:382-393, DOI 10.1086/320642, astro-ph/0008389

Pons JA, Steiner AW, Prakash M, Lattimer JM (2001b) Evolution of ProtoNeutron Stars with Quarks. Physical Review Letters 86:5223-5226, DOI 10. 1103/PhysRevLett.86.5223, astro-ph/0102015

Prakash M, Bombaci I, Prakash M, Ellis PJ, Lattimer JM, Knorren R (1997) Composition and structure of protoneutron stars. Phys. Rep.280:1-77, DOI 10.1016/S0370-1573(96)00023-3, nucl-th/9603042

Qian YZ, Woosley SE (1996) Nucleosynthesis in Neutrino-driven Winds. I. The Physical Conditions. Astrophys. J. 471:331, DOI 10.1086/177973, arXiv: astro-ph/9611094

Raffelt GG (1996) Stars as laboratories for fundamental physics : the astrophysics of neutrinos, axions, and other weakly interacting particles

Raffelt GG (2001) Mu- and Tau-Neutrino Spectra Formation in Supernovae. Astrophys. J. 561:890-914, DOI 10.1086/323379, astro-ph/0105250

Reddy S, Prakash M, Lattimer JM (1998) Neutrino interactions in hot and dense matter. Phys. Rev. D58(1):013009, DOI 10.1103/PhysRevD.58.013009, arXiv: astro-ph/9710115

Reddy S, Prakash M, Lattimer JM, Pons JA (1999) Effects of strong and electromagnetic correlations on neutrino interactions in dense matter. Phys. Rev. C59:28882918, DOI 10.1103/PhysRevC.59.2888, astro-ph/9811294

Roberts LF (2012) A New Code for Proto-Neutron Star Evolution. ArXiv e-prints 1205.3228

Roberts LF, Woosley SE, Hoffman RD (2010) Integrated Nucleosynthesis in Neutrino-driven Winds. Astrophys. J. 722:954-967, DOI 10.1088/0004-637X/ 722/1/954, 1004.4916

Roberts LF, Reddy S, Shen G (2012) Medium modification of the charged-current neutrino opacity and its implications. Phys. Rev. C86(6):065803, DOI 10.1103/ PhysRevC.86.065803, 1205.4066

Roberts LF, Shen G, Cirigliano V, Pons JA, Reddy S, Woosley SE (2012) Protoneutron star cooling with convection: The effect of the symmetry energy. Phys Rev Lett 108:061,103, DOI 10.1103/PhysRevLett.108.061103, URL http:// link.aps.org/doi/10.1103/PhysRevLett.108.061103

Rrapaj E, Roggero A, Holt JW (2015) Mean field extrapolations of microscopic nuclear equations of state. Submitted to Phys Rev C 1510.00444

Scholberg K (2012) Supernova Neutrino Detection. Annual Review of Nuclear and Particle Science 62:81-103, DOI 10.1146/annurev-nucl-102711-095006, 1205. 6003

Steiner AW, Prakash M, Lattimer JM (2001) Diffusion of neutrinos in protoneutron star matter with quarks. Physics Letters B 509:10-18, DOI 10.1016/ S0370-2693(01)00434-8, astro-ph/0101566

Steiner AW, Lattimer JM, Brown EF (2013) The Neutron Star Mass-Radius Relation and the Equation of State of Dense Matter. Astrophys. J. Lett.765:L5, DOI 10. 1088/2041-8205/765/1/L5, 1205.6871 
Sumiyoshi K, Suzuki H, Toki H (1995) Influence of the symmetry energy on the birth of neutron stars and supernova neutrinos. Astron. \& Astrophys.303:475, arXiv:astro-ph/9506024

Tews I, Krüger T, Hebeler K, Schwenk A (2013) Neutron matter at next-tonext-to-next-to-leading order in chiral effective field theory. Phys Rev Lett 110(3):032,504, DOI 10.1103/PhysRevLett.110.032504, 1206.0025

Thorne KS (1981) Relativistic radiative transfer - Moment formalisms. Mon. Not. Roy. Astro. Soc.194:439-473

Wilson JR, Mayle RW (1988) Convection in core collapse supernovae. Phys. Rep.163:63-77, DOI 10.1016/0370-1573(88)90036-1

Woosley SE, Weaver TA (1995) The Evolution and Explosion of Massive Stars. II. Explosive Hydrodynamics and Nucleosynthesis. Astrophys. J. Sup.101:181, DOI 10.1086/192237

Woosley SE, Hartmann DH, Hoffman RD, Haxton WC (1990) The nu-process. Astrophys. J. 356:272-301, DOI 10.1086/168839

Woosley SE, Wilson JR, Mathews GJ, Hoffman RD, Meyer BS (1994) The r-process and neutrino-heated supernova ejecta. Astrophys. J. 433:229-246, DOI 10.1086/ 174638

Woosley SE, Heger A, Weaver TA (2002) The evolution and explosion of massive stars. Reviews of Modern Physics 74:1015-1071, DOI 10.1103/RevModPhys.74. 1015 